\title{
Fever in Common Infectious Diseases
}

\section{Core Messages}

$\checkmark$ Infection of the respiratory tract is the most common reason for seeking medical advice and hospital admission in children. A viral upper respiratory tract infection (URTI) is the most common infection of the respiratory tract.

>In developing countries, acute respiratory infection remains a leading cause of childhood mortality, causing an estimated 1.5-2 million deaths annually in children younger than 5 years of age.

> In developed countries, viruses are responsible for most upper and lower respiratory tract infections, including pharyngitis and pneumonia.

> Although the degree of fever cannot differentiate between viral and bacterial diseases, high fever is associated with a greater incidence of serious bacterial diseases such as pneumonia or meningitis.

> Worldwide, diarrheal disease is the leading cause of childhood deaths under 5 years of age.

> If the fever does not have an evident source, urinary tract infection (UTI) should be considered, particularly if the fever is greater than $39.0^{\circ} \mathrm{C}$ and persists for longer than $24-48 \mathrm{~h}$.

) Widespread vaccinations against bacteria causing meningitis, such as Hib, and vaccines against meningococci and pneumococci have dramatically reduced the incidence of meningitis.

> A child with fever and nonblanching rash should be promptly evaluated to exclude meningococcal diseases.

> Young children with malaria may present with irregular fever and not with typical paroxysms of fever, occurring particularly in early falciparum infection or as a consequence of previous chemoprophylaxis, which modifies the typical pattern of fever. 


\section{1}

\section{Acute Upper Airway Infection}

An upper airway infection is the most common infection in children, accounting for half to two-thirds of all childhood infections. This term includes viral URTI, tonsillopharyngitis, otitis media (OM) and epiglottic diseases.

\subsection{1 \\ Viral Upper Respiratory Tract Infection (URTI)}

URTI is an exceedingly frequent infection characterized by nasal obstruction and discharge, fever, throat irritation, malaise, headache, and cough. The initial watery nasal discharge is followed rapidly by mucopurulent nasal discharge, which does not necessarily indicate bacterial infection. The combination of innate immunity and maternal immunoglobulin-G (IgG), transferred during the last trimester of pregnancy, provides some protection against the organisms causing URTI. It has been estimated that young children may have as many as 12 respiratory infections per year if he or she attends nursery, 9 infections per year if a sibling attends school, and 6 or 7 per year if the child and a sibling are not at school [1].

Well over 100 viruses are known to cause respiratory tract infection, such as rhinoviruses, influenza A and B, coronaviruses, parainfluenza 1, 2, and 3, adenoviruses, and respiratory syncytial viruses (RSV). Infection may result from inhalation, self-inoculation to the nasal mucosa, or airborne inoculation to the conjunctival mucosa. Children tend to have greater concentrations of viruses in the nasal secretion and shed them for longer periods of time than adults. Viremia is less common and the infection is usually restricted to the mucosa, including the sinuses and Eustachian tube.

Inflammatory mediators such as interferons (INF), rather than the virus itself, are responsible for many symptoms associated with cold. Injection of INF- $\alpha$ to volunteers has been found to cause fever, malaise, headache, and myalgia [2].

Fever in URTI has been studied [3,4], and the findings in affected children were:

- Fever was present in $50 \%$ of older children and in $90 \%$ of infants and young children.

- The degree of fever was not helpful in differentiating viral (mean degree $39.2^{\circ} \mathrm{C}$ ) from bacterial infection (mean degree $\left(39.3^{\circ} \mathrm{C}\right)$.

- A temperature greater than $39^{\circ} \mathrm{C}$ was recorded in $59 \%, 40^{\circ} \mathrm{C}$ or greater in $12 \%$, and $40.5^{\circ} \mathrm{C}$ or greater in $3 \%$.

- None of the children studied had a temperature exceeding $41^{\circ} \mathrm{C}$, suggesting that a child with such a high degree of fever is likely to have another cause.

- High fever $\left(>39.5^{\circ} \mathrm{C}\right)$ was associated with influenza A virus infections, occurring in more than $50 \%$ of children.

- Adenovirus infection caused fever exceeding $40^{\circ} \mathrm{C}$ in about $20 \%$, while fever in rhinovirus infection was usually absent or mild. 
- Fever associated with respiratory virus infection may last for 3-5 days. A prolonged duration of fever is, however, common. Fever lasting 5 days or longer occurred in $37 \%$ of children with this infection. The longest duration of fever ( $>7$ days) may occur in association with adenovirus. The shortest duration of fever was associated with parainfluenza 2 viruses.

The knowledge that viruses can cause high and/or prolonged fever allows the physician to withhold antibiotic treatment and extensive investigations in children with prolonged fever who appear generally well, and common serious infections (e.g., UTI) have been excluded. In differential diagnosis, conditions mimicking URTI include the following:

- Allergic rhinitis. There is often a family history of atopy and a history of nasal symptoms with exposure to potential allergens, and the symptoms tend to be persistent. Nasal eosinophilia, increased serum IgE level, and the finding of possible allergens in skin prick tests or blood may confirm the diagnosis.

- Streptococcal throat infection. Children are usually older than 5 years. Fever tends to be higher than that in URTI. Nasal symptoms are usually absent.

- Sinusitis should be considered in a child with purulent nasal discharge and fever (usual range $38-39^{\circ} \mathrm{C}$ ), localized pain and tenderness, mucosal erythema, and headache, whereas a higher fever with chills may suggest an extension of the infection beyond the sinuses.

For a febrile child with an URTI, the following recommendations may be useful:

- No specific therapy is indicated for most children.

- Symptomatic relief may sometimes be obtained with antipyretics in a febrile child with pain, excessive myalgia, or malaise. Paracetamol in a dose of $10-15 \mathrm{mg} \mathrm{kg}^{-1}$ may be administered at $4 \mathrm{~h}$ intervals.

- Antibiotics have no place in uncomplicated cases and should be avoided, as should antihistamines and cough suppressants.

- Nasal decongestants are rarely required, except perhaps for those infants with feeding or sleeping difficulty caused by the nasal obstruction.

- Vitamin C has been advocated for common cold, but its value is unproven.

- Amantadine is licensed for prophylaxis and treatment of influenza A but it is no longer recommended (see National Institute for Health and Clinical Excellence (NICE) guidance). Oseltamivir and zanamivir reduce replication of influenza A and B viruses by inhibiting viral neuraminidase. They are most effective for the treatment of influenza if started within a few hours of the onset of symptoms. Oseltamivir is licensed for use within $48 \mathrm{~h}$ of the first symptoms, while zanamivir is licensed for use within $36 \mathrm{~h}$ of the first symptoms. In otherwise healthy individuals they reduce the duration of symptoms by about 1-1.5 days. Oseltamivir is not licensed for use in children under 1 year because of concerns about neurotoxicity but it may be used under specialist supervision if the child is seriously ill. For further information on the treatment of influenza (see NICE guidance).

- There is limited data to suggest that antibiotics may reduce the duration of fever in children with Influenza. If there is any effect, this is likely due to a reduction in secondary bacterial infection. Figure 5.1 shows the proportions of children with virus infections who recovered with or without antibiotics. 

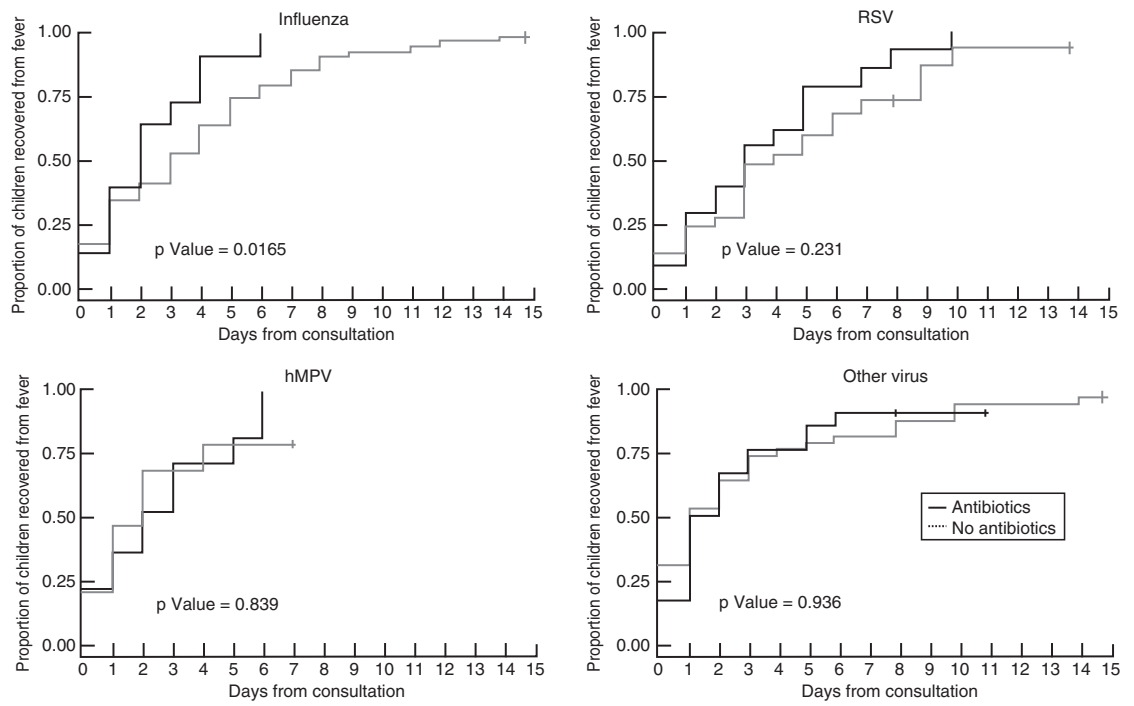

Fig. 5.1 Time to resolution of fever (days from consultation) according to virus detected and antibiotic prescribing. $\mathrm{hmpv}=$ human metopneumovirus, RSV = respiratory syncytial virus (obtained from Arch. Dis. Child. 2007; 92; 594-597; originally published online 16 Mar 2007; doi:10.1136/adc.2007. 116665)

\section{1 .2}

\section{Tonsillopharyngitis}

Although most cases of tonsillopharyngitis are caused by viral agents, group A- $\beta$-haemolytic streptococci (GABHS) are the most common bacterial cause. Less common causes are pneumococci and other groups of $\beta$-hemolytic streptococci.

Streptococcal tonsillopharyngitis may begin abruptly with fever, malaise, sore throat, headaches, and abdomoinal pain. On examination, the tonsils are oedematous and hyperemic. There may be purulent exudates confined to the tonsils. The uvula is red and swollen, and the anterior cervical lymphnodes are enlarged and tender. There is usually an absence of conjunctivitis, coryza, or cough.

Cytokines, particularly interleukin-6 (IL-6) and tumor necrosis factor- $\alpha$ (TNF- $\alpha$ ), play an important role in the pathogenesis of the inflammatory process of tonsillopharyngitis. High fever correlates with high levels of these two cytokines.

Diagnosis of streptococcal tonsillopharyngitis is established by throat culture and a more than twofold rise in antistreptolysin titers between two serum samples taken 2 weeks apart. Rapid antigen detection and polymerase chain reaction (PCR) tests are useful for providing rapid results.

The differential diagnosis of streptococcal tonsillitis is:

- Scarlet fever results from certain strains of haemolytic streptococci producing an erythrogenic toxin. The rash is an erythematous punctiform eruption that blanches on 
pressure and spares the area around the mouth. Initially the tongue has a thick white cover, which develops in a few days into the typical strawberry tongue. Apart from the rash and the tongue, there is essentially no difference between streptococcal tonsillitis and scarlet fever. Fever in both conditions usually ranges from 39 to $40.5^{\circ} \mathrm{C}$ peaking on the second day of illness. Without treatment, the temperature usually subsides on the fifth day, whereas penicillin therapy causes a rapid normalization of temperature within $12-24 \mathrm{~h}$.

- Peritonsillar abscess is a rare suppurative complication of tonsillitis causing a toxic appearance, fluctuant peritonsillar mass, and asymmetric deviation of the uvula.

- Gingivostomatitis is usually caused by herpes simplex infection in infants and small children. It is characterized by irritability, anorexia, and fever, which is usually in the range $38.5-39.5^{\circ} \mathrm{C}$ (rarely in the range of $40-40.5^{\circ} \mathrm{C}$ ). The child has painful oral vesicles that soon rupture. Submaxillary lymphadenitis may occur. The disease is self-limited and lasts for about a week.

- Coxsakie viurs A may cause herpangina. The initial temperature ranges from normal up to $41^{\circ} \mathrm{C}$; the temperature tends to be higher in younger children. Other features include headache and vomiting. Throat inspection reveals discrete punctuate vesicles, surrounded by erythematous rings on the soft palate, anterior pillars, and uvula.

- Diphtheria, which develops insidiously, has a grey, thick membrane that bleeds easily if removed. The associated fever in diphtheria is typically low grade. Frequently, there is a pharyngeal erythema and congestion (with or without tonsillar exudates). Anterior cervical adenitis is commonly present.

Penicillin eradicates streptococci from the throat, bringing the symptoms, including fever, rapidly under control and effectively preventing suppurative and nonsuppurative complications. Macrolides are adequate antibiotics in case of penicillin allergy. Paracetamol is used for pain and fever.

\subsection{3 \\ Otitis Media (0M)}

$\mathrm{OM}$ is one of the commonest infections in children, particularly during the first 2 years of life, affecting about $60 \%$ of all children. At risk are those who attend day care centers and those whose parents smoke at home. The infection usually arises from a URTI, which spreads to the middle ear through the short and straight Eustachian tube. Streptococcus pneumoniae accounts for most of the bacteria recovered by tympanocentesis. In neonates and infants during the first 6 months of life, the most frequent bacteria are E. coli, Klebsiella, group B streptococci and staphylococcus aureus. Viruses are currently the most common cause of OM.

OM often presents with a sudden febrile illness characterized by:

- High fever, irritability, pain in the ear, and a prompt response to antibiotics. A temperature of $<39^{\circ} \mathrm{C}$ occurs in about $25 \%$ and a temperature of $>39^{\circ} \mathrm{C}$ in about $75 \%$. The highest fever is recorded in children less than 2 years of age. 
- History of or the presence of a URTI and sudden rise of fever.

- Rapid response of the fever following antibiotic therapy. Only 4\% of children persist with fever lasting longer than $48 \mathrm{~h}$. Persistent fever may suggest a viral cause, resistant bacteria, unsuitable antibiotic, or a complication of OM.

- High cytokine levels, which correlate with the degrees of fever. In OM and middle ear effusion, high levels of cytokines exist, including IL-1 and TNF- $\alpha$.

The absence of fever suggests a more insidious variety of OM characterized by mild lassitude and irritability. Conductive hearing loss is often present with this variety.

Complications include perforation of the drum, mastoiditis, chronic otitis, cholesteatoma, facial paresis, increased intracranial pressure (causing bulging of the fontanelle in infants), meningitis, brain abscess, or lateral sinus thrombosis.

The use of antibiotics is controversial. The majority of cases of OM are caused by viral infection. If antibiotics are prescribed, Amoxicillin or a macrolide are sensible choices. The introduction of pneumococcal vaccines will help to further reduce the number of bacterial middle ear infections. The use of antihistamine, ear drops, or decongestant is controversial. Analgesics are often needed to reduce the pain. For a bulging tympanic membrane, or if the response to antibiotics is not prompt, myringotomy may rarely be considered for aspiration of fluid.

\subsection{4}

\section{Infectious Mononucleosis}

Infectious mononucleosis (IM) is an acute viral infection caused by Epstein-Barr virus (EBV). The symptoms, laboratory findings, and complications are shown on Table 5.1.

Table 5.1 Features and complications of clinical data of infectious mononucleosis ${ }^{\mathrm{a}}$

\begin{tabular}{|c|c|c|c|c|c|}
\hline Physical signs & $\%$ & Laboratory findings & $\%$ & Complication & $\%$ \\
\hline Fever & 100 & EB-IgM & 100 & Pneumonia & 3 \\
\hline Lymphadenopathy & 80 & Monospot test & 98 & Hemolytic anaemia & 3 \\
\hline Pharyngitis & 80 & High transaminases & 90 & Agranulocytosis & 0.1 \\
\hline Splenomegaly & 50 & $>50 \%$ lymphocytes & 50 & Thrombocytopenia & 0.1 \\
\hline $\begin{array}{l}\text { Rash } \\
\text { Palatal petechiae } \\
\text { Exanthem }\end{array}$ & $\begin{array}{l}50 \\
10\end{array}$ & & & $\begin{array}{l}\text { Neurological } \\
\text { Guillian-Barre syndrome } \\
\text { Meningoencephalitis } \\
\text { Transverse myelitis }\end{array}$ & 1.5 \\
\hline Hepatomegaly & 20 & & & & \\
\hline Jaundice & 5 & & & $\begin{array}{l}\text { Other rare complications: } \\
\text { Ruptured spleen } \\
\text { Myocarditis }\end{array}$ & \\
\hline Airway obstruction & $1-3.5$ & & & $\begin{array}{l}\text { Pericarditis } \\
\text { Arthritis } \\
\text { Nephritis }\end{array}$ & \\
\hline
\end{tabular}

a Modified from [5] and [6] 
A prodromal period of 3-5 days with malaise, fatigue, and headache may precede the onset of fever. Most (80\%) patients will have fever symptoms pharyngeal and lymphadenopathy (pharyngeal form) and 20\% present with fever alone (typhoidal form) [5]. Fever may last 4 days to 2 or 3 weeks (mean duration 1 week) peaking on the fifth day of illness [6]. The pattern of fever is frequently intermittent, with a usual range between 38.5 and $39.5^{\circ} \mathrm{C}$, but rarely higher than $40^{\circ} \mathrm{C}$. IM may presents as

- an asymptomatic infection occurring during early childhood;

- a typical IM triad of fever, pharyngitis, and lymphadenopathy occurring mainly in adolescents and young adults;

- a case of pyrexia of unknown origin (PUO), with fever as the only sign of the disease;

- a complication listed in Table 5.1;

- a cytomegalovirus mononucleosis characterized by prolonged fever and liver and haematological changes similar to those observed in Epstein-Barr infection. Heterophile antibodies are always absent. Pharyngitis is uncommon;

- Tonsillopharyngitis not responding to antibiotic administration or as an extensive rash following the use of ampicillin;

- EBV infection in association with a range of malignancies including Burkitt's lymphoma, nasopharyngeal carcinoma, and, in immunocompromised individuals, lymphoproliferative diseae [7].

Leukocytosis in the range of $10,000-20,000$ is frequent. Absolute lymphocytosis $(>50 \%)$ and atypical lymphocytes are usual findings. Tests to demonstrate heterophile antibodies (positive in more than $80 \%$ ) have been superseded by several rapid slide tests (Monospot). IgM is positive in almost $100 \%$. PCR for detection of EBV is now routine to aid diagnosis.

Therapy. IM is usually self-limiting, requiring only symptomatic treatment. Paracetamol is used to reduce the fever and pain. Steroids, usually given for two weeks, do not influence the extent or the duration of fever, and they are indicated mainly for impending airway obstruction. Hyperplasia of the lymphoid tissue in Walder's ring may occasionally cause severe airway obstruction and will respond to steroids. Emergency tonsillectomy and adenoectomy or tracheostomy are rarely required. Patients, particularly those with splenomegaly, should avoid excessive activity and trauma to minimize the risk of splenic rupture. Splenic rupture and neurological complications are rare but account for most fatalities.

\subsection{5}

\section{Acute Upper Airway Obstruction}

Viral croup (laryngotracheobronchitis) is a common cause of upper respiratory tract obstruction of the subglottic area. It is characterized by inspiratory stridor, cough, hoarse voice, and a variable degree of respiratory distress. Although symptoms often appear alarming, the infection is a benign, self-limiting illness, which usually persists for 2-6 days. Parainfluenza viruses account for about $75 \%$ of all isolates. Other pathogens include influenza A and B, adenovirus, and mycoplasma pneumonia. The attack rate is highest in the second year of life, and male children are predominately affected.

Onset is sudden (usually at night) with loud stridor and barking cough, preceded by a URTI. The severity of croup is assessed by a scoring system (Table 5.2). 


\begin{tabular}{lll} 
& Mild-moderate & Severe \\
- Stridor & Minimal & severe \\
- Chest retraction & None & obvious \\
- Air entry & Normal & decreased \\
- Cyanosis & absent & present \\
- Level of consciousness & Normal & disorientated, \\
& & drowsy \\
\hline
\end{tabular}

Table 5.2 Scoring system of severity for children with croup

Variable degrees of fever are present in about $40 \%$ the patients with croup, ranging between 38 and $39^{\circ} \mathrm{C}$ (mean $38.7^{\circ} \mathrm{C}$ ). Children with spasmodic croup are usually afebrile. White blood cell count (WBC) and C-reactive protein (CRP) are often normal or slightly increased. Guidelines to manage a child with croup include:

- Most children with croup recover rapidly with minimal medical intervention. Children with mild croup and minimal or no respiratory distress can be managed at home. If hospitalization is required, the mother should, whenever possible, be with the child to minimize stress.

- Moderate to severe croup with stridor with or without respiratory distress may benefit from nebulized budesonide ( $2 \mathrm{mg}$ ), a single dose of parenteral or oral dexamethasone $0.6 \mathrm{mg} \mathrm{kg}^{-1}$ and oxygen if there is hypoxia $\left(\mathrm{O}_{2}\right.$-saturation $\left.<94 \%\right)$.

- Nebulized epinephrine (adrenalin) is effective in producing dramatic effects in alleviating the airway obstruction in severe croup.

- Although humidification is commonly used, trials have not shown this to greatly influence the clinical course of croup.

- Intubation is based on clinical grounds and supported by the presence of signs of respiratory failure as evident by hypercarbia.

Spasmodic croup is another entity of unknown etiology. Onset is always at night. The characteristic presentation occurs in a child who previously has been well without associated upper respiratory infection and who awakens at night with sudden dyspnea, croupy cough, and inspiratory stridor. Fever is usually absent.

Bacterial tracheitis is a bacterial infection (Staphylococcal aureus or H. influenzae) of the tracheal mucosa, often producing thick purulent exudates. This infection usually begins as a viral croup but progresses rapidly with high fever, toxicity, and worsening respiratory distress.

Epiglottitis is an acute bacterial infection characterized by marked swelling of the glottis and arytenoids area. Septicemia caused by $H$. influenzae type B is present in most cases. Epiglottitis is rarely seen nowadays following Hib vaccines. The infection has an abrupt onset with high fever, respiratory distress, dysphagia, drooling, irritability, restlessness, anxiety, and a thick muffled voice. In a report of 100 consecutive admissions of children with epiglottitis, fever was noted in 88 , with a range from 39 to $40.5^{\circ} \mathrm{C}$, [8] and a mean of $39.1^{\circ} \mathrm{C}$.

Differentiating epiglottitis from viral croup may be difficult. Epiglottitis is now very rare. Patients appear very unwell, with higher degrees of fever and respiratory distress, and there is usually leukocytosis and high CRP. 


\section{2 \\ Acute Lower Airway Infection}

\subsection{1}

\section{Bronchiolitis}

A clear distinction between bronchiolitis and bronchitis in the first 2 years of life is difficult and of no therapeutic significance. Both are preceded by URTI. The diagnosis of bronchiolitis is made in the presence of a history of a URTI followed by acute onset of respiratory distress with cough, breathlessness, wheezing, tachypnea, and clinical signs of chest inflation, occurring during a winter epidemic of bronchiolitis. RSV is the most common etiological agent. Peak age is 4-6 months. Pre-existing chronic lung disease, congenital heart disease, immunodeficiency, prematurity, parental smoking, apnea, and infection in very early infancy predispose to severe illness and occasional death.

Information on the incidence of fever in bronchiolitis or on its relationship to clinical severity of bronchiolitis is limited. In a study of 90 children with bronchiolitis [9], fever (defined as a single recording of $>38^{\circ} \mathrm{C}$ or two successive recording $>37.8^{\circ} \mathrm{C}$ ) was present in 28 infants (31\%). Febrile children had a longer mean hospital stay and a more severe clinical course compared to those who were afebrile (Table 5.3).

In infants with bronchiolitis, hypoxia is common, and as many as $40 \%$ to $50 \%$ of cases require oxygen suppliment. A rise of body temperature results in an increase in energy expenditure of about $10 \%$ for each $1{ }^{\circ} \mathrm{C}$ rise in temperature. These changes are accompanied by an increase in oxygen consumption of $10-12 \%$ for every $1{ }^{\circ} \mathrm{C}$ rise in temperature [10]. The low incidence of fever in bronchiolitis may be due to low interferon production. Although interferon is known to be a potent endogenous pyrogen, this cytokine is significantly low during acute RSV bronchiolitis [11].

The mainstay of treatment is adequate oxygenation and hydration. Hypoxia, as measured by pulse oximetry, requires oxygen administration. Naso-gastric tube feeding or intravenous fluid is often required in moderate or severe cases to maintain fluid balance, to replace fluid loss from insensible sweating or tachypnea, and to minimize aspiration. Inhalation of a beta-2 agonist (salbutamol) or an anticholinergic agent (Ipratropium) is effective if there are signs of bronchospasms (wheezing or rhonchi on auscultation). Antibiotics are of no value unless the disease is complicated by bacterial infection. Corticosteroids, antihistamine, cough suppressants, and expectorants are also of unproven value. Ribavirin can be effective in reducing the shedding of virus and is used in immunocompromised patients.

Table 5.3 Summary of clinical data of 90 children hospitalized with bronchiolitis [9]

\begin{tabular}{llll} 
Group & $\begin{array}{l}\text { Length of stay in } \\
\text { days (Mean) }\end{array}$ & \multicolumn{2}{c|}{ Clinical severity } \\
\cline { 3 - 4 } & & Severe & Mild \\
\hline Febrile $(n=28)$ & $4.2(1-13)$ & $20(71 \%)$ & $8(28.6 \%)$ \\
Afebrile $(n=62)$ & $2.7(1-10)$ & $18(29 \%)$ & $44(71 \%)$ \\
$p$-value & $<0.005$ & & $<0.005$ \\
\hline
\end{tabular}


Palivizumab, an antibody directed against the virus, is recommended to prevent RSV in high risk individuals such as immunocompromised children.

\subsection{2}

Asthma

Asthma is defined as reversible obstructive airway disease characterized by bronchospasm, mucosal edema, and mucosal plugging. The airway obstruction is unevenly distributed throughout the lungs, leading to ventilation-perfusion imbalance and hypoxia. There is increased airway responsiveness to various stimuli, including respiratory viruses, house dusts, exercise, air pollutants, cigarette smoking, and drugs. Cytokines enhance eosinophil differentiation and maturation as well as endothelial adherence and activation. IgE synthesis is dependent on IL-4.

The clinical hallmarks of the disease are paroxysms of expiratory wheezing with prolonged expiratory phase, unproductive cough, and dyspnea. The predominant asthma type in school-age children (6-16 years) is the classic atopic variant that is associated with allergy problems as evidenced by the strong correlation with serum $\operatorname{IgE}$ levels and with skin-test reactivity to allergens. By contrast, asthma in children aged 1-5 years is characterized by recurrent, transient wheeze triggered by viral colds, a type previously termed as wheezy bronchitis and now as preschool viral wheeze. Physical examination reveals varying degrees of tachycardia, tachypnea, use of accessory muscles of respiration, and rhonchi on auscultation. Somnolence, fatigue, diminished wheezing, and breath sounds usually signal respiratory failure.

Markers of asthma severity include an admission to hospital in the previous 12 months, less privileged social class, parental smoking, and the frequency of prior and recent respiratory infections. Environmental factors such as climate and air pollution have been found to influence the prevalence and severity of asthma. Higher exposure rates to cockroaches and dust mites have also correlated with asthma severity. There are three stages of asthma severity:

- During early stage, hypoxia causes increased minute ventilation, a fall in $\mathrm{Pco}_{2}$ and normal or elevated $\mathrm{pH}$ (respiratory alkalosis).

- Moderate asthma is associated with increased hypoxia, normal $\mathrm{Pco}_{2}$, and $\mathrm{pH}$.

- In severe asthma, hypercarbia, low $\mathrm{pH}$, and respiratory/metabolic acidosis (respiratory failure) ensue as a result of respiratory muscle fatigue, hypoxia, and anaerobic cellular metabolism producing lactic acidosis.

Fever is not a frequent finding in acute exacerbations of asthma. It was recorded in only $18.8 \%$ on admission of 202 patients, mostly younger than 5 years of age (Table 5.4) [12]. Viruses are the most common cause of lower respiratory tract infection during the first few years of life and they can induce a febrile response.

The reasons why most asthmatic children are afebrile may be due to:

- Tachypnoea, which accompanies asthma, increases heat loss through evaporation.

- Reduced interferon production has been reported in children with bronchiolitis and asthma. Interferon is known to be a potent endogenous pyrogen capable of inducing fever. 
Table 5.4 Clinical data of 202 children with asthma [12]

\begin{tabular}{|c|c|c|c|c|}
\hline & \multirow{2}{*}{$\begin{array}{l}\text { Mean age in } \\
\text { months (range) }\end{array}$} & \multirow{2}{*}{$\begin{array}{l}\text { Mean stay in days } \\
\text { in hospital (range) }\end{array}$} & \multicolumn{2}{|c|}{ Asthma severity } \\
\hline & & & Severe & Mild \\
\hline \multicolumn{5}{|l|}{$<5$ years } \\
\hline Febrile $=27$ & $24(12-42)$ & $1.7(1-4)$ & 1 & 26 \\
\hline Afebrile $=70$ & $31(12-58)$ & $1.9(1-9)$ & 5 & 65 \\
\hline$p$-value & 0.012 & 0.484 & 0.603 & \\
\hline $95 \% \mathrm{CI}$ & $1.6-12.7$ & -0.3 to 0.7 & $\mathrm{n} / \mathrm{a}$ & \\
\hline \multicolumn{5}{|l|}{$>5$ years } \\
\hline Febrile $=11$ & $113(78-172)$ & $1.4(1-2)$ & 1 & 10 \\
\hline Afebrile $=94$ & $118(60-180)$ & $2.2(1-6)$ & 18 & 76 \\
\hline$p$-value & 0.700 & 0.065 & 0.688 & \\
\hline $95 \% \mathrm{CI}$ & $19.1,28.3$ & $0.65,15$ & $\mathrm{n} / \mathrm{a}$ & \\
\hline
\end{tabular}

- Cytokines implicated in the pathogenesis of asthma, such as IL-5, IL-8, IL-4, and eosinophil cationic protein (ECP), are not known to be potent pyrogens, and their presence is unlikely to induce fever in asthma [13].

In this study [12], the severity of asthma was found to be inversely related to the degree of fever: children with severe asthma were usually afebrile, and mildly asthmatic children were often febrile. Similar observations were made at the turn of the last century when patients were noted to obtain a temporary relief of their asthma in association with fever. Subsequently various methods (diathermy) were used to treat fever [14]. It is possible that

- fever as a response to infection can limit the spread of infection by enhancing the host defense mechanisms to eliminate the viruses;

- as airway inflammation is a cardinal feature of asthma, cortisol, a potent glucocorticoid, is known to be elevated in febrile illness and could play a role as an endogenous antiinflammatory agent [15].

Laboratory investigations such as full blood count, CRP and erythrocyte sedimentation rate (ESR) are of little value in asthma. Leukocytosis is common in the absence of bacterial infection. A chest X-ray is rarely indicated, unless the diagnosis is uncertain, in patients with fever $>39^{\circ} \mathrm{C}$ and in case of severe asthma. Measurement of oxygen saturation is always indicated when a child is admitted to hospital. For older children, measurement of the peak flow is important and should be repeatedly performed. Allergy skin tests (to detect IgE antibody in the skin to inhalants such as pollens and house dust mites) and the radioallergosorbent test (RAST, detecting IgE to various allergens in the serum) are often performed, but they do not seem to be of great diagnostic or therapeutic value.

The most important principle of therapy is rapid reversal of the airway obstruction. Nebulized $\beta-2$ agonists remain the first line of treatment. Theophyllin may be added in severe asthma. Children with mild-moderate asthma who are not taking adequate fluids by mouth and all children with severe asthma should have intravenous (i.v.) fluid therapy. Corticosteroids are recommended for patients with acute severe asthma, but their effects are slow. Steroid therapy reduces symptoms and bronchial hyper-responsiveness 
and is currently widely used as an anti-inflammatory agent in asthma. Oxygen should be administered in all cases with hypoxia, delivered through a face mask to bring the oxygen saturation to $>92 \%$. Inhaled steroids are used prophylactically for patients who have more frequent asthma attacks and who have not improved on beta- 2 agonist alone.

\section{3}

\section{Pneumonia}

In 1900, pneumonia, called by Osler "the captain of the men of death", was the most common cause of death, annually killing more than 200 people per 100,000 in the United States. By 1940 , pneumonia was relegated to third, with approximately 70 deaths per 100,000 [16]. In children, pneumonia is common, but its true incidence is not established owing to the lack of an accepted clinical definition of pneumonia. Peak incidence occurs between 6 months and 5 years. Factors that increase the risk of pneumonia include malnutrition, parental smoking, immunosuppression, low socioeconomic status, and prematurity.

The lungs are not only involved in gas exchange but also in mediating host defense. This includes nonimmunological defense mechanisms (such as lysozyme secretion by macrophages) and immunological defense mechanisms (such as activation of macrophages and $\mathrm{B}$ and $\mathrm{T}$ lymphocytes). The alveolar macrophages respond to activation by exogenous pyrogens (such as endotoxin released by Gram-negative bacteria) by releasing potent inflammatory mediators, including IL-1, TNF, and IL-8. This leads to a febrile response, accumulation of neutrophils at the site of infection, and inhibition and repair of tissue injury. IL-8 is particularly important for neutrophil chemotaxis.

Diagnosis of pneumonia is based on the following features:

- Symptoms include fever, cough, dyspnea, tachypnea, grunting and nasal flaring, and referred pain. Lower lobe pneumonia may cause lower abdominal pain mimicking acute appendicitis. Upper lobe pneumonia may cause meningism (increased cerebrospinal fluid (CSF) pressure, but CSF is otherwise normal), causing suspicion of meningitis.

- Findings include inspiratory rales and bronchial breathing on auscultation. Tachypnea ( $>40 / \mathrm{min}$ aged $>1$ year, $>50 / \mathrm{min}$ aged $2-12 \mathrm{~m}$ and $>60 / \mathrm{min}$ aged $<2 \mathrm{~m}$ ) is the WHOdefined criterion to diagnose pneumonia.

- Wheezing, cough and fever may occur with mycoplasma infection.

- Chest X-ray is diagnostic but is often of limited value in distinguishing bacterial and viral. The presence of effusion and/or lobar consolidation suggests bacterial etiology.

Isolation of the pathogens causing pneumonia is usually not possible in practice. Bacterial culture from the pharyngeal area or expectorated sputum is unreliable. However, pathogens can be identified by:

- blood culture (positive in $10 \%$ of cases with bacterial pneumonia);

- serum or urine counterimmuno-electrophoresis as a rapid and more sensitive technique than blood culture for the detection of bacterial antigens;

- culture of aspirated pleural effusion;

- high IgM, such as mycoplasma pneumoniae; 
- respiratory secretion for rapid virus antigens (e.g., RSV, parainfluenza);

- serological tests showing fourfold rise of antibody titers;

- PCR, which is increasingly being used.

Marked leukocytosis, sometimes exceeding $40,000 \mathrm{~mm}^{-3}$ (leukomoid reaction) is very suggestive of bacterial pneumonia, particularly pneumococcal or $H$. influenzae pneumonia. Although inflammatory markers (WBC, CRP) are usually normal in viral pneumonia, mild leukocytosis with a left shift in the differential count may occur, particularly in influenza pneumonia.

Fever is the most common symptom of pneumonia in children older than 1 month of age. Reports $[17,18]$ on fever and pneumonia have indicated the following:

- Of the 100 febrile children with pneumonia, a temperature of $>40^{\circ} \mathrm{C}$ occurred in 45 , while the remaining 55 children had a fever of $<40^{\circ} \mathrm{C}$.

- Fever was present in all children with $H$. influenzae pneumonia, with a mean temperature on admission of $39.9^{\circ} \mathrm{C}(\mathrm{H}$. pneumonia is rarely seen nowadays).

- The onset of pneumococcal pneumonia was usually abrupt with a temperature of $39.5-40.5^{\circ} \mathrm{C}$. The highest fever, however, tended to be with staphylococcal infection. (A temperature of $41^{\circ} \mathrm{C}$ is not an unusual finding.)

- The likelihood of pneumonia increased with increasing duration of fever longer than 3 days, that is, during a febrile URTI. A study of 711 children with pneumonia from 13 hospitals in England found that CRP, chest X-ray changes, or pyrexia were not associated with increased severity of the disease.

- In children $<3$ years old, a combination of $>38.5^{\circ} \mathrm{C}$, chest recession, and a respiratory rate of $>50$ per minute indicates pneumonia. Dyspnea is a more reliable sign of pneumonia in older children (The British Thoracic Society guidelines).

\subsection{1}

\section{Pneumonia in Newborn Infants}

Pneumonia during the neonatal period is usually caused by organisms acquired during or before delivery, mainly Group B streptococci. The amniotic fluid may be infected, or the mother is an asymptomatic carrier of these organisms. Predisposing factors are prolonged rupture of membrane, prolonged labor, or an infected, febrile mother. The infection by these bacteria is mainly due to low levels of opsonizing antibodies directed at the polysaccharides of the organism, impaired function of the lung macrophages, and polymorphonuclear leukocytes. Pneumonia may also accompany a generalized intrauterine infection by cytomegalovirus toxoplasmosis, listeria, or rubella virus. Chlamydia trachomatis is classically an afebrile pneumonia with a dry cough and increasing tachypnea. Conjunctivitis is present in about $50 \%$ of cases.

The newborn infant with pneumonia usually presents with signs of respiratory distress with tachypnea and grunting. Body temperature is often normal. If the mother has been febrile before delivery, an increased temperature may be detected in the infant in the first few hours of life owing to the constant temperature gradient between mother and infant during pregnancy. 


\subsection{2}

\section{Pneumonia at Age 1 Month to 4 Years}

During this age, the rate of viral pneumonia is high, particularly in children around six months of age. RSV remains the most common cause of pneumonia in industrialized countries. Febrile pneumonias are commonly caused by RSV, influenza A and B, parainfluenza type B, and adenoviruses, while afebrile pneumonias are usually due to C. trachomatis, cytomegalovirus, or Mycoplasma hominis. Commonly, an URTI precedes the onset of pneumonia.

In developing countries, the causes and patterns of pneumonias are affected by malnutrition, poor housing, lack of early medical attention, and immunization. Pneumococci, streptococci, coliforms, H. influenzae and staphylococci are the more common causes of pneumonia with high mortality.

Staphylococcal pneumonia is a rather rare cause of pneumonia occurring sometimes as a complication of the influenza virus infection. Its presentation is with shaking chills and high fever $>40^{\circ} \mathrm{C}$, pallor, tachypnea, abdominal distension, and, rarely, cyanosis. The diagnosis should be suspected in any child younger than 1 year of age who appears ill and does not respond rapidly to conventional antibiotics such as penicillin and ampicillin. Chest X-ray shows multiple nodules, which undergo cystic formation (pneumatocele) and empyema.

\subsection{3}

\section{Pneumonia at the Age of $>4$ Years}

Pneumococcal and Mycoplasma pneumonia are the most frequently identified organisms, whereas viruses are less common at this age. In pneumococcal pneumonia, patients have often flu-like symptoms for several days before the onset of pneumonia, which begins by an abrupt onset of rigor and high fever. The cough becomes intense and is usually accompanied by chest pain. The sputum is classically rusty in color due to alveolar hemorrhage, but this is seldom seen nowadays in children.

Mycoplasma pneumoniae is characterized by insidious onset of fever, headaches, and abdominal pain, followed by cough. Transient skin rash is found in about $10 \%$ of cases. Mycoplasma pneumonia may also present with similar clinical and radiological features of pneumococcal and staphylococcal infection. In contrast to these infections, however, children with M. pneumonia appear well despite the extent of the X-ray lesions

Fever is present in more than $90 \%$ of patients. In a study of 66 children with mycoplasma infection [19], the temperature distribution was as follows: temperature $<38^{\circ} \mathrm{C}$ was present in $22 \%$ of patients; $38.3-38.9^{\circ} \mathrm{C}$ in $30 \% ; 39.4-40^{\circ} \mathrm{C}$ in $44 \%$, and $>40.6^{\circ} \mathrm{C}$ in $4 \%$.

The diagnosis of M. pneumonia is difficult, but it may be made by a combination of the following:

- History of unresponsiveness to penicillin or amoxicillin

- A fourfold rise in antibody titer or a single titer of 128 or more

- IgM antibodies 
- Serum cold-agglutinins in $50-70 \%$ of the cases

- Chest X-ray which is not diagnostic but commonly showing peribronchial and perivascular interstitial infiltrates

- A possibly high CRP or ESR and a normal WBC count.

About 5\% may develop neurological complications, such as encephalitis, meningitis, cerebellar ataxia, focal neuropathy, or cerebral infarction. Other complications are hemolytic anemia, arthritis, rash (popular, vesicular, erythema multiform) myocarditis, pericarditis, and interstitial nephritis.

\subsection{4}

\section{Pneumonia at Any Age}

Aspiration pneumonia may occur subsequent to aspiration of secretion from the oropharynx in weak or neurologically impaired children (e.g., in preterm infants, cerebral palsy), in children with tachypnea (e.g., bronchiolitis), or following inhalation or accidental ingestion of kerosene or aspiration of gastric acid. The child presents with dyspnea, tachypnea, subcostal recession, cough, wheezing, and cyanosis. Children are usually afebrile with aspiration pneumonia, with possible exception of kerosene pneumonia, which is often associated with fever of $38-39.5^{\circ} \mathrm{C}$. Chest X-ray shows infiltrates usually involving the right upper lobe in infants and right lower lobe in older children.

Pneumocystis jiroveci pneumonia (Previously known as Pneumocystis carinii, the organism responsible for Penumocystis carinii pneumonia, PCP) occurs almost exclusively in patients who are immunocompromised, including those who are receiving immune-suppressive drugs for malignancy or organ transplantation, or HIV infection. About $85 \%$ of patients with HIV develop PCP during the course of their illness. Unlike most infectious complications in cancer patients, PCP may occur while the patient is in remission from the primary cause. Clinical manifestations include fever, cough, cyanosis, marked tachypnea with intercostals retraction and a paucity of physical signs of pneumonia. Among 1251 children with malignancies, PCP was identified in 51 (4.1\%) [20]. Fever was the first sign of abnormality and occurred in almost all patients with, or shortly preceding, tachypnea. The extent of the fever varies from mild to severe.

The diagnosis is suggested by a chest X-ray showing a hazy, bilateral alveolar infiltration. Sputum examination and bronchoalveolar lavage (BAL) can identify PCP in most cases. The diagnosis is confirmed by detecting PCP by immunohostochemistry or PCR or by histological or cytological demonstration of thick-walled cysts, as obtained by BAL or from percutaneous transthoracic needle aspiration of the lung.

Antibiotic therapy of pneumonia depends on the age of the child and likelihood of the causative agent. Neonates are treated with penicillin and gentamicin. Older children respond to second and third-generation cephalosporins or co-amoxiclav. Suspected cases of staphylococcal pneumonia should receive anti-staphylococcal agent such as flucloxacillin. Patients with mycoplasma pneumonia usually respond well to macrolides. The treatment of choice for patients with PCP is trimethoprim-sulfamethoxazole (TMP-SMX) $20 \mathrm{mg} \mathrm{kg}^{-1}$ per day. 


\section{4 \\ Gastroenteritis}

Worldwide, diarrheal disease is the leading cause of death under 5 years of age [21]. Data collected from 276 surveys on diarrhea in 60 countries between 1981 and 1986 have shown that one-third of all deaths in children below 5 years of age is caused by diarrhea. Approximately 1.5 billion diarrheal episodes and 4.6 million deaths in children occur per year (or 12,600 deaths per day), accounting for $21-29.3 \%$ of all childhood deaths [22,23]. In the absence of diarrheal diseases, the total infant and child mortality in developing countries would not differ significantly from that of developed countries [24].

In developing countries, bacterial (Escherichia coli, salmonella, shigella, campylobacter, and Yersinia enterocolitica) and parasitic (Entamoebia histolytica, Giardia lamblia, Crptosporidium species) pathogens are the major causes of gastroenteritis, particularly in summer months (Table 5.5). In Tanzania, diarrhegenic E. coli (35.7\%) were the predominant enteropathogens [25]. In a recent study from Vietnam, stool pathogens were identified in $67.3 \%$, of which rotavirus and diarrheagenic E. coli were the most common isolates [26].

In a study from the United States, 372 stool specimens from children with diarrhea grew bacteria in 176 (47\%): Shigella toxin-producing E. coli (22.2\%), Salmonella species (22.2\%), Campylobacter species (14.2\%), Shigella species (8\%), and Yersinia enterocolitica (1.1\%). Rotavirus was detected in $22.8 \%$ [27].

Fever or hyperthermia is common in both bacterial and viral gastroenteritis. High fever is commonly present in many bacterial causes (e.g., Shigella, Salmonella, Shiga toxin-producing E. coli). Fever is often absent or of low grade in other diseases (e.g., enteropathogenic E. coli, cholera). Other febrile conditions that cause diarrhea are shown in Table 5.6. Bacteria or viruses acting as exogenous pyrogens can cause fever by inducing endogenous pyrogens, which raises the hypothalamic thermoregulatory set point. Clinical and laboratory findings that can differentiate bacterial from viral etiology of acute gastroenteritis are shown in Table 5.7.

Dehydration, the most common cause of hyperthermia, leads to cutaneous vasoconstriction and decreased sweating, causing an increase in body temperature. In hypernatremic dehydration, an increase in sodium pump activity needed to offset the high extracellular sodium concentration may further raise the body temperature. High sodium levels may

\begin{tabular}{lll} 
Bacteria & Virus & Parasites \\
\hline Salmonella & Rotavirus & Giardia lamblia \\
Shigella & Adenovirus & Entamoebia histolytica \\
E. coli & Other viruses & \\
Campylobacter jejuni & \\
Yersinia & \\
Vibrio cholerae & \\
Other bacteria & \\
\hline
\end{tabular}

Table 5.5 Major enteropathogenic agents in children with gastroenteritis 
Table 5.6 Febrile non-enteritis diseases that cause diarrhoea

\begin{tabular}{ll} 
Conditions & Diagnostic clue \\
\hline Intussusception & $\begin{array}{l}\text { Intermittent, colicky abdominal pain, abdominal mass, } \\
\text { cherry-red stoots }\end{array}$ \\
HUS & $\begin{array}{l}\text { Bloody diarrhea, abdominal pain, renal failure, haemolysis } \\
\text { Appendicitis }\end{array}$ \\
Neuroblastoma & Abdominal mass \\
Primary Immunodeficiency & Associated recurrent infections \\
HIV-infection & Commonly associated with thrush, recurrent infections, \\
& weight loss \\
Kawasaki disease & Lymphadenopathy, conjunctivitis, rash, fever $>5$ days \\
Addison's disease & $\begin{array}{l}\text { Diarrhea occurs in chronic adrenal insufficiency, abnormal } \\
\text { electrolytes, pigmentation, adynamy }\end{array}$ \\
Crohn's disease & Associated weight loss, anaemia, high CRP \\
\hline
\end{tabular}

HUS hemolytic uremic syndrome

Table 5.7 Factors likely to predict the etiology of acute gastroenteritis

\begin{tabular}{ll} 
Bacterial etiology & Viral etiology \\
\hline Fever $>39^{\circ} \mathrm{C}$ & No fever or low-grade fever \\
Presence of bloody stools & No bloody stools \\
Summer months & Winter months \\
High CRP, WBC, IL-6 & Normal or mildly elevated CRP, WBC, IL-6 \\
Hyponatremia is common & Hyponatremia is uncommon \\
Increased WBC in stool & None to few WBC in stool \\
High serum TNF- $\alpha$ & Low serum TNF- $\alpha$ \\
\hline
\end{tabular}

$C R P$ C-reactive protein; $W B C$ white blood cell count; $I L$ interleukin; $T N F$ tumor necrosis factor.

also act directly upon the hypothalamus to increase the set point. For every $1{ }^{\circ} \mathrm{C}$ increase of body temperature there is an increase in insensible water loss of $10 \%$. Table 5.8 shows typical water losses based on caloric expenditure of $100 \mathrm{kcal} \mathrm{kg}^{-1}$ per day for an infant weighing $10 \mathrm{~kg}$ body weight.

\subsection{1}

\section{Bacterial Gastroenteritis}

Bacterial gastroenteritis is caused either by secretory pathogens (such as cholera, which causes watery diarrhea through colonization and adherence to the small bowel mucosa) or invasive pathogens (such as Shigellae, which cause inflammatory cell exudates in the distal bowel and/or colon). Secretory pathogens are likely to cause severe diarrhea. Invasive 


\begin{tabular}{ll} 
Source of water loss & $\begin{array}{l}\text { Approximate water loss } \\
\left(\mathbf{m l ~ k g}^{-1} \text { per day }\right)\end{array}$ \\
\hline $\begin{array}{l}\text { Insensible } \\
\text { Skin }\end{array}$ \\
$\quad 30$ \\
Respiratory & $15-20$ \\
Sensible & \\
$\quad$ Stool & 10 \\
Urine & $50-60$ \\
Total & $105-120^{\mathrm{a}}$ \\
\hline
\end{tabular}

Table 5.8 Typical water losses per $100 \mathrm{kcal}$ of energy expended for a healthy $10 \mathrm{~kg}$ child

a The above average calculation. The sum of insensible water loss (average $50 \mathrm{ml} \mathrm{kg}^{-1}$ per day) with $16 \mathrm{ml} \mathrm{kg}^{-1}$ per day subtracted for endogenous water for oxidation produces $34 \mathrm{ml} \mathrm{kg}^{-1}$ per day. The addition of $66 \mathrm{ml} \mathrm{kg}^{-1}$ per day urinary loss would produce $100 \mathrm{ml}$ $\mathrm{kg}^{-1}$ per day fluid requirement

organisms may cause watery or grossly bloody diarrhea with cramps and tenesmus, but severe diarrhea is infrequent.

Salmonellae are Gram-negative rods with over 1400 known species. The most common serotypes are $S$. typhimurium, $S$. enteritidis, and $S$ infantis. In industrialized countries nontyphoidal salmonellae (NTS) infection is more common. This is usually a self-limiting and benign disease, and invasion beyond the gastrointestinal tract occurs in only about $5 \%$ of patients. In many African countries bacteremia is a major cause of death, and NTS account for $20-50 \%$ of cases, ranking second only to pneumococcal pneumonia as the leading bacterial cause of child mortality [28]. In the United States it is between 800,000 and 3,700,000 [29]. Most human infections occur in late summer and early autumn and are caused by the ingestion of contaminated food (meat, poultry products, eggs) or water. Increased susceptibility to the infection occurs in children with sickle cell anemia, impaired cellular immunity, and achlorohydria.

About 12-48h following ingestion of contaminated food, the onset is abrupt with nausea, fever, and crampy abdominal pain, followed by loose, watery diarrhea, occasionally containing mucus, blood, or both. The illness is indistinguishable from Shigella infection. Vomiting is a not a striking feature in salmonellosis.

Whereas salmonellosis in older children is usually a self-limiting disease requiring no antibiotic therapy, there is a significant incidence of bacteremia (range 15-45\%) and meningitis in infants younger than 3 months. Bacteremia may occur in the absence of fever in this age group. The absence of fever usually excludes bacteremia in older children.

Endotoxin is a complex lipopolysaccharide structure that constitutes the outside portion of the cell wall of Salmonella. Endotoxin releases IL-1 from macrophage into the circulation, accounting for the fever and other systemic manifestations of the disease. Cytokines are responsible for the symptoms and development of the protective mechanisms in the disease. Mean serum concentrations of TNF- $\alpha$, TNF- $\gamma$, and IL-12 were found to be increased during the acute phase of the disease and in those patients with early bacterial clearance compared with those of healthy controls and nonclearance patients [30]. 
In a study from Finland [31] comprising 102 children with salmonella gastroenteritis, 15 had a fever $>40^{\circ} \mathrm{C}, 66$ had a fever of $38-39.9^{\circ} \mathrm{C}$, and 21 had a temperature of $<37.9^{\circ} \mathrm{C}$. There was a significant correlation between the degree of fever and the duration of organism excretion: a fever of $>40^{\circ} \mathrm{C}$ had the shortest and no fever the longest duration of excretion (Table 5.9). Fever therefore appears to have a favorable prognostic influence on the duration of salmonella excretion. The gastrointestinal tract acts a major barrier against the potentially noxious substances, such as microbes. Immunological defenses include secretory IgA, macrophage, and activated T lymphocytes in the Peyer patches and lamina propria. Fever is beneficial to the infected host by enhancing macrophage and T-and B-cell activity.

Typhoid fever includes infection with $S$. typhi and $S$. paratyphi A, B and C, and rarely S. choleraesuis, S. heidlberg, and S. typhimurium. The incidence of typhoid fever in the United States is 0.2 cases per 100,000 population, with a case fatality rate of $1.3 \%$ [32].

Elevated proinflammatory pyrogenic cytokines, particularly TNF- $\alpha$ and IL-6, are responsible for the prolonged fever, which is characteristic of the disease. High serum levels of these cytokines have been linked to disease severity. A high level of IL-6 suggests poorer response to antibiotic therapy and its decline correlates with successful therapy. Vigorous antipyretic use may lead to shock.

In older children, presentation of a typical case follows the following steps:

Onset is insidious with fever (without shaking chills) present in all patients, and is associated with headache, cough, and abdominal pain.

Symptoms then gradually increase over 2-3 days. The child is often constipated, nauseated, and anorexic.

The temperature continues to rise in a stepwise fashion to reach $40-41^{\circ} \mathrm{C}$. In young children the onset of fever is more often abrupt, then becoming sustained or intermittent. The stepwise pattern of fever is less common. In all ages, fever may continue for many days despite successful antibiotic therapy, and the child does not become afebrile until the end of the therapy. At the end of the first week, patients remain febrile with hot, dry skin, abdominal tenderness, hepatosplenomegaly, and relative bradycardia. Roseate spots may be detected in about $20-40 \%$, characterized by a few discrete popular erythematous lesions confined to the anterior chest and abdomen. Delirium, convulsion, meningeal irritation, psychosis, and ataxia may be noted.

Table 5.9 Fever on admission and duration of bacterial excretion after salmonella gastroenteritis in 102 children [31]

\begin{tabular}{lllll} 
Degree of fever $\left({ }^{\circ} \mathbf{C}\right)$ & No of children & \multicolumn{2}{c}{$\begin{array}{c}\text { Duration of salmonella excretion } \\
\text { (weeks) }\end{array}$} \\
\cline { 3 - 5 } & & Range & Mean & Mean (SD) \\
\hline (A) $>400$ & 15 & $0-10$ & 0 & $1.9(2.9)$ \\
(B) $38-38.9$ & 66 & $0-18$ & 3 & $4.1(4.0)$ \\
(C) $<37.9$ & 21 & $2-60$ & 7 & $11.7(15.1)$ \\
\hline
\end{tabular}

$S D$ standard deviation.

$p$ Value: (A) vs. (B), $p=0.160$; (B) vs. (C), $p=0.0011$; (A) vs. (C), $p=0.0001$ 
If untreated with antibiotics, fever remains continuous at $39-40.5^{\circ} \mathrm{C}$ for $2-3$ weeks before abating slowly.

By the end of 2 weeks, perforation or hemorrhage (in about $5 \%$ ) may occur owing to typhoid ulceration and defects in coagulation. This serious complication is associated with $50 \%$ mortality.

Typhoid bacilli persist indefinitely in the bile passage in about $3-5 \%$ who recover from the infection.

Laboratory findings include leucopenia, anemia, thrombocytopenia, and increased serum aspartate transaminase (SGOT). Elevated agglutination titers of $\mathrm{O}$ and $\mathrm{H}$ antigens at 1:160 are significant. The diagnosis is based on isolation of $S$. typhi or other salmonella strains from blood or bone marrow culture.

Shigellae are gram-negative rods with worldwide distribution. Humans are the principal host for shigellosis (bacillary dysentery). Most patients are under 5 years of age; the infection is rarely seen in infants under 6 months of age.

Four serotypes are known: Shigella flexneri, S. dysenteriae, S. sonnei, and S. boydii. The first two species are more common in developing countries, whereas $S$. boydii and $S$. sonnei usually cause a self-limiting febrile illness in developed countries. Shigella must penetrate the mucosa in order to cause dysentery.

The disease onset is usually acute with fever and malaise, often progressing to dysentery consisting of cramps, tenesmus, and frequent stools composed largely of blood and mucosa. Severe dehydration is not a typical feature of the infection. High fever is common. Of 57 children with Shigella gastroenteritis, $27(47 \%)$ had a fever greater than $40^{\circ} \mathrm{C}$ at presentation, $21(37 \%)$ had a fever between 38 and $40^{\circ} \mathrm{C}$, and the remaining $9(16 \%)$ were afebrile [33]. In a recent study from Thailand, out of 80 children with shigellosis, the mean age of infection was 3.6 years. Fever was present in $77.6 \%$, vomiting in $44.8 \%$, and seizures in $27.6 \%$. Shigella sonnei was the commonest species isolated (62.8\%) [34].

Complications include toxic megacolon, protein-losing enteropathy, hyponatremia due to inappropriate antidiuretic hormone secretion, disseminated intravascular coagulation, renal failure, hemolytic uremic syndrome and bacteremia. Shigella bacteremia occurs in $4.0 \%$ of patients. Neurological symptoms, particularly convulsion, are among the most frequent extraintestinal manifestations of shigellosis occurring with or without evidence of the production of Shiga's toxin (neurotoxin). Death can occur in children with poor nutritional state.

Amoebic dysentery, caused by Entameba histolytica, may cause colitis simulating shigellosis. Virulence of E. histolytica depends on the trophozoites being able to bind to colonic epithelium. The infection tends to run a more chronic course with intermittent watery or semiformed diarrhea (containing blood and mucosa) without or with a low-grade fever. Young children tend to present with acute symptoms similar to cases with Shigella infection. Liver abscess may occur a few months after the intestinal infection, causing discomfort over the liver, intermittent fever with chills and sweats, and weight loss. Findings suggestive of amoebic liver abscess include an elevated right diaphragm, hepatomegaly, and a history of colitis. The diagnosis of amoebiasis is confirmed by demonstration of E. histolytica in a stool (motile trophozoites during the diarrhea, cyst if the diarrhea is not present) or in tissues. The indirect hemagglutination (HA) test and enzyme-linked immunosorbent assays (ELISA) are positive in almost all patients with amoebic liver abscess and in the majority of those with intestinal infection. 
E. coli cause either non-bloody diarrhoea (e.g., enterotoxigenic $E$. coli $=E T E C$, enteropathogenic $E$. coli $=E P E C$ ) or bloody diarrhea (e.g., Shigatoxin-producing $E$. coli $=$ STEC, enteroinvasive $E$. coli $=$ EIEC). Enteroaggregative E.coli $($ EAggEC) cause significant fluid loss and dehydration, but bloody stools are relatively infrequent.

- ETEC produce enterotoxins that cause copious watery diarrhea in developing countries. In its severe form, the illness resembles cholera, and is responsible for high mortality among young children. It is an uncommon cause of diarrhea in industrial countries, but it is the most common cause of traveler's diarrhea. EPEC infection was in the past a common cause of outbreaks of infantile diarrhea in industrialized countries, usually occurring in neonates and young children $<2$ years of age. Since the 1970 s, the infection has been reported less frequently, and the severity of the illness has lessened in children of these countries. It can cause protracted diarrhea.

- EIEC and STEC produce Shiga toxins, causing a dysentery-like diarrhea and hemolytic uremic syndrome (HUS) by the strain 0157:H7.

Fever occurs frequently in the range of $38-40^{\circ} \mathrm{C}$. Fever, very often low grade, is reported in only one-third of patients infected with EIEC and STEC. EPEC usually does not cause systemic manifestations because the organisms remain confined to the bowel lumen. Fever was found in only 5 of the infected 49 children from Addis Ababa [35].

Campylobacter enteritis is an important cause of enteritis in both developed and developing countries. C. jejuni is the most important species. The enteritis is a zoonosis, and a man-to-man transmission is unusual. Raw cow's milk and incompletely cooked poultry meat have caused most of outbreaks in the United Kingdom. Contaminated water is another cause of outbreaks particularly in developing countries.

Infection is usually self-limiting in industrialized countries, lasting 2-4 days, following an incubation time that averages 5 days. Children present with an acute illness accompanied by fever, diarrhea, and bloody stools in about $90 \%$ of cases. Abdominal pain occurs almost universally, but vomiting is mild and occurs in about $30 \%$. In a study from Canada, all 32 children with this infection who were older than 12 weeks developed fever up to $40.5^{\circ} \mathrm{C}$, whereas all 5 children who were younger than 12 weeks remained afebrile. In a study from Iraq (202 children with diarrhea; $13.86 \%$ detection rate of C. Jejuni), fever was detected in (82.14\%) [36].

Yersinia enterocolitica is an anaerobic, Gram-negative bacillus that causes an infection mostly in cooler climates such as Scandinavia and Canada. Serotype O:3 is the most common isolate. Transmission of $Y$. enterocolitica to humans occurs from ingestion of contaminated foods (particularly contaminated pork), water, and milk.

Presentation is characterized by bloody diarrhea in about one-third of patients. The fever is usually mild, ranging between 38 and $39^{\circ} \mathrm{C}$. The associated abdominal pain sometimes mimics appendicitis (pseudo-appendicitis). The disease is usually mild, although it can be prolonged ( 1 day to 3 weeks). A Canadian study of 181 children with Yersinia infection (45) reported that diarrhea occurred in $98 \%$, fever in $88 \%$, abdominal pain in $64.5 \%$, and vomiting in $38 \%$ [37].

Rare complications are arthritis, erythema nodosum, intestinal perforation, diffuse ulceration, iliocolic intussusception, peritonitis, glomerulonephritis, meningitis, and perimyocarditis. The infection can occur as septicemia, and patients then have high fever, toxic appearance, and confusion. 


\subsection{2 \\ Viral Gastroenteritis}

Rotavirus infection is responsible for $30-60 \%$ of all cases of dehydration and diarrhea in young children (peak age 3-15 months) in both developed and developing countries. The infection is prevalent in winter months. Approximately 125 million cases of rotavirus diarrhea occur annually in developing countries, leading to an estimated 800,000-900,000 deaths a year and $25 \%$ of all deaths due to diarrheal diseases [38]. Although viremia is rare in healthy individuals, this was reported in $67 \%$ of immunocompetent children with rotavirus diarrhea [39].

Nosocomial acquired outbreaks of rotavirus have occurred in newborn nurseries and pediatric hospital wards. The virus can be detected in oropharyngeal aspirates with or without diarrhea. Spread occurs via the fecal-oral route. The virus is shed in feces in high concentration, which allows its easy identification by electron microscopy.

There is often a preceding or accompanying URTI or OM. Within 2 days of exposure, there is fever and vomiting, which last 1-3 days, and usually preceding the onset of watery diarrhea, which lasts 4-7 days. Dehydration occurs as a result of marked fecal fluid loss.

Most children with rotavirus diarrhea have fever. A study from Finland [40] reported that $14 \%$ of 336 infants had fever of $39-40.2^{\circ} \mathrm{C}$ and $65 \%$ had less than $39^{\circ} \mathrm{C}$. Many cytokines, particularly IL-6, IL-10, and INF- $\gamma$, play an important role in the pathogenesis of as well as protection against rotavirus re-infection. IL-6 is elevated in children with fever. A vaccine is now available, although its use is not widespread as yet.

Enteric adenovirus. Several studies have shown that adenovirus is second to rotavirus as the most common cause of viral gastroenteritis, occurring commonly during the first year of life. The infection was identified in $8.6 \%$ of 900 pediatric inpatients with diarrhea, serotypes 40 and 41 being the most common isolates [41]. In contrast to enteric adenoviruses, other respiratory adenoviruses are not associated with diarrhea. Watery diarrhea is the most common presentation, usually followed by 1-2 days of vomiting. Illness typically lasts 5-12 days (mean 9 days). The duration of the diarrhea usually lasts longer compared to that caused by rotavirus. Severe dehydration is less common compared to rotavirus infection.

A low-grade fever for 1-3 days is commonly recorded with adenovirus enteritis. A Canadian study [42] of 127 children with adenovirus enteritis found that $41 \%$ of them had a rectal temperature of $>38^{\circ} \mathrm{C}$. The range of body temperature was $36.2-40.8^{\circ} \mathrm{C}$ (mean $38^{\circ} \mathrm{C}$ ). The average duration of fever was 1.6 days (range 1-30 days).

The outcome of adenovirus gastroenteritis is generally good. Adenovirus is now more frequently diagnosed (due to PCR) in immunocompromised patients and is an important cause of mortality.

Other viruses. Norfolk virus and Norwalk-like viruses are major causes of small and large outbreaks of winter vomiting in older children and adults with or without diarrhea. These outbreaks occur commonly in recreational camps, communities, or schools in the United States. Presentation is similar to that of other types of viral gastroenteritis and includes anorexia, malaise, and fever and abdominal cramps, followed within $48 \mathrm{~h}$ by vomiting and watery diarrhea. Symptoms usually last 2-3 days, and full recovery is the usual out- 
come. Astrovirus can also cause gastroenteritis. The infection is frequently asymptomatic in the newborn infants.

Treatment. Breast milk is the best prophylaxis against gastroenteritis, and exclusively breast-fed children remain remarkably free of severe diarrhea in developed and developing countries.

The standard treatment of all diarrheal diseases is the replacement of fluid and electrolyte loss. This is best accomplished by oral rehydration solution (ORS), which has revolutionized the management of diarrheal diseases in developing countries. This is safe, cheap, convenient to use, and superior to i.v. fluids because it can be started early at home. The sugar-electrolyte mixture recommended by the WHO contains (mmol $1^{-1}$ water) sodium 90 , chloride 80 , potassium 20 , sodium bicarbonate 30 , glucose 111 ; with an osmolality of 331 mosmol $1^{-1}$. A hypotonic solution with a sodium concentration of as low as 50-60 $\mathrm{mmol} \mathrm{l}^{-1}$ and an osmolality of $224 \mathrm{mosmol} \mathrm{l}^{-1}$ has been shown in Finland to have clinical advantages over the standard ORS [43]. A systematic review found that rice-based ORS compared with standard ORS reduced the 24-h stool volume [44]. Intravenous electrolyte-glucose solution should be used for children with moderate to severe dehydration and persistent vomiting.

Antibiotic therapy is usually not required for patients with gastroenteritis because it does not affect the clinical course in most cases. Severe systemic manifestations associated with bacterial gastroenteritis (notably Shigella, campylobacter, Yersinia, and cholera) probably require antibiotics. Infants less than 3 months of age with salmonella gastroenteritis should be treated with an antibiotic, such as a third-generation cephalosporin or a quinolone depending on the regional resistance pattern. Patients with typhoid fever and E. histolytica should also receive antibiotic treatment.

\section{5}

\section{Viral Hepatitis}

Hepatitis occurs as a result of a variety of causes, including viruses (hepatitis viruses, EBV, cytomegalovirus), bacteria (leptospirosis), parasitic infection (amoebiasis), and drugs.

\subsection{1}

\section{Hepatitis A}

Hepatitis A (HAV) is a highly contagious infection, spreading mostly by fecal-oral contact from person to person.

The clinical features are usually mild, and most infected children have an anicteric illness with flu-like symptoms or gastroenteritis with lethargy, nausea, vomiting, abdominal pain, and anorexia. Clinical findings often reveal a tender and enlarged liver. Splenomegaly is present in about $20 \%$ of cases. About $99 \%$ of children recover completely from the infection. Fulminant hepatic failure may occur in the remaining $1 \%$. Chronic hepatitis or cirrhosis is not part of the HAV infection. 
Fever, usually low grade between 38 and $39^{\circ} \mathrm{C}$, is found in about $40 \%$ of cases. The low incidence of fever in hepatitis is probably due to impaired production of IL-1, which increases slightly during the first week of illness, reaching a peak during the second and third week, and thereafter decreasing to a normal level. The impaired production of IL-1 does not appear to correlate with the severity of liver disease.

Diagnosis rests on detection of the specific IgM, which is a marker of recent infection. It is usually positive before the onset of jaundice, peaking at 1 week and is undetectable 4-8 weeks later. IgG anti-HAV indicates previous exposure and is detectable approximately 1 week later than IgM, and persists for years as a sign of immunity. High transaminase enzymes are characteristic of the disease. These enzymes are elevated during the anicteric phase of the illness and usually persist for a few weeks. Serum bilirubin and alkaline phosphatase are mildly or moderately elevated. Prothrombin time is usually normal.

Standard immunoglobulin preparations administered within 2 weeks of exposure have proved effective in preventing hepatitis A. Vaccine HAV is effective.

\subsection{2}

\section{Hepatitis B Virus}

Transmission of this virus usually occurs via the parenteral route or any bodily secretion or fluid. Children exposed to multiple blood transfusions are at high risk of contracting the virus. The incubation period for Hepatitis B (HBV) infection ranges from 6 weeks to 6 months (mean 90 days). The HB surface antigen (HBsAg) appears during the incubation period several weeks before clinical or biochemical illness develops, and is usually undetectable after 6 months. The core antigen ( $\mathrm{HBcAG}$ ) and e antigen (HBeAG) are other antigens of HBV associated with greater infectivity.

Neonates are at high risk if the mother has acute hepatitis or carries HbsAg (chronic carrier) at delivery. Viral acquisition may follow swallowing of maternal blood during delivery, rarely via the transplacental route, or through ingestion of breast milk. Most infants born to HBsAg-positive mothers remain asymptomatic for months and years.

Clinical manifestations are usually absent or mild without evidence of fever. The vast majority of children ( $>90 \%$ ) infected with this virus develop a chronic carrier state, and less than $5 \%$ develop hepatitis. Children are at risk of developing hepatocellular carcinoma and should therefore be regularly monitored with serial ultrasound scan and serum $\alpha$-fetoprotein. Patients have substantial abnormalities of cell-mediated immunity and cytokine production, including a decreased production of TNF- $\alpha$.

In HBV infection in older children, prodromal symptoms may include urticaria and arthralgia, which precede a spectrum of clinical presentations ranging from acute viral hepatitis, severe or fulminant hepatitis, chronic persistent hepatitis, and chronic active hepatitis to the asymptomatic chronic carrier state . 


\section{6 Urinary Tract Infection}

Urinary Tract Infection (UTI) is a common cause of an acutely febrile illness in children affecting $7 \%$ of girls and $2 \%$ of boys. The infection is mainly caused by ascending fecal bacteria from the perineum to the bladder. UTI is frequently the result of sepsis during the first three months of life, occurring more commonly in males. Known predisposing factors for UTI include maternal febrile UTI, congenital malformation of the urinary tract, urolithiasis, indwelling urinary catheter, constipation, and uncircumcised males. The principal sequence of UTI is vesico-ureteric reflux found in $30 \%$ of acute cases, which may lead to chronic renal failure and/or hypertension in adults.

The most common organisms are:

- Uropathogenic E. coli, which contain lipopolysaccharide, lipoprotein, and proteoglycan. By attachment of the bacteria to the urinary tract, these substances are capable of inducing an inflammatory response and fever.

- Less common etiological agents include Proteus mirabilis, Klebsiella pneumoniae, enterococci, and Staphylococcus epidermidis.

The proinflammatory cytokines IL-6 and IL-8 play an important role in the inflammatory process of UTI. Serum and urine IL- 6 and urine IL- $1 \beta$ positively correlate with fever in UTI.

UTI presents in one of the following ways:

- High fever, rigor, vomiting, meningism and abdominal discomfort, loin pain, and tenderness;

- Fever without apparent source (FWAS), occurring mainly in infants. The prevalence of UTI in FWAS varies between 4 and 13\% (mean of 10\%);

- As sepsis with or without fever, occurring in $30 \%$ of neonates, $21 \%$ of infants aged $1-2$ months, and $5.5 \%$ of infants older than 3 months of age [45];

- Febrile seizure as the first symptom of an underlying UTI;

- Dysuria, frequency, urgency, or dribbling, occurring in older children, particularly in girls. The condition is usually afebrile, termed as lower UTI;

- Asymptomatic (without fever) bacteriuria is common in school girls (1-2\%) but the infection is of little clinical or prognostic significance.

Diagnosis of UTI rests on the following findings:

- A febrile child without a focus whose urine may harbour the infection;

- Positive nitrite and leukocytes in the urine dipsticks, which are very suggestive of the diagnosis while the negative result of these two indicators virtually excludes it. A positive urinalysis is defined as 5 or more WBC per high power field;

- Urine culture as the ultimate tool to confirm or refute the diagnosis. UTI is diagnosed if the urine shows a colony count of 100,000 colonies per milliliter of a single bacterial species. Suprapubic puncture is important for accurate diagnosis during infancy. In older children midstream urine sample is sufficient;

- Leukocytosis $>15,000$, high CRP ( $>40 \mathrm{mg} \mathrm{l}^{-1}$ ) to support the diagnosis. CRP is particularly valuable when fever has been present for more than $12 \mathrm{~h}$. IL-6 is a useful diagnostic tool for early recognition of UTI; 
Once UTI is confirmed, recent guidelines suggest that a renal ultrasound scan should be arranged early in case of severe or recurrent infections. Micturating cystourography (MCUG) is currently less commonly performed than previously and should be reserved (along with DMSA isotope scan) for atypical presentation, or recurrent infections occurring in infancy.

Fever in UTI has the following significance:

- It is the leading complaint for seeking medical attention.

- High and prolonged fever is associated with high incidence of UTI.

- UTI is unlikely and insignificant without fever in children older than 2 months.

- In 1025 febrile children younger 2 months from the United States, uncircumcised male infants had a higher rate of UTI (21.3\%), compared with female (5\%) and circumcised males $(2.3 \%)$. Infants with high fever $>39.0^{\circ} \mathrm{C}$ had a higher rate of UTI $(16.3 \%)$ than those infants with lower degrees of fever (7.2\%) [46].

Therapy. Systematic reviews of 23 studies (3295 children) showed no significant differences in persistent renal damage or duration of fever between oral antibiotics (a second-generation cephalosporin, cefixime, or co-amoxiclav) and short courses (2-4 days of i.v. therapy followed by oral therapy [47].

In conclusion, the diagnosis of UTI should be considered in every febrile child, particularly when the fever is without a focus and of a duration longer than $24-48 \mathrm{~h}$. A delay in diagnosis and treatment increases the risk of scarring.

\section{7 \\ HIV Infection}

By 2007, an estimated 33 million people are living with human immunodeficiency virus (HIV)/AIDS, with more than 2 million people newly infected and more than 25 million deaths since 1981 [48]. HIV is affecting millions of children worldwide. The vast majority of children are infected through vertical transmission from mother to child (about 95\%). Typical presenting symptoms are fever, asthenia, failure to thrive, prolonged diarrhoea, recurrent infections, and lymphadenopathy.

Fever is very common in HIV infection, occurring in $85 \%$ (49\%) and is caused by:

- The HIV infection itself

- Concurrent bacterial infection of gastrointestinal or respiratory system;

- Immune reconstitution syndrome (IRS). This is a transient deterioration or emergence of new manifestations (such as high fever, worsening of central nervous system (CNS) lymphadenitis lesions) of an opportunistic infection occurring after the initiation of antiretroviral therapy (ART). The syndrome also occurs after initiating anti-TB treatment in patients already on ART;

- Secondary infections (such as tuberculosis);

- Drug fever, commonly associated with ART;

- Unknown causes of fever, which may present as a case of pyrexia of unknown orgin (PUO). 
The rate of concomitant secondary infections among the HIV-infected children has been estimated to be 3 times higher than the rate in the non-HIV-infected children [50]. The incidence of fever is higher with a co-infection such as TB. Children with fever $>39.0^{\circ} \mathrm{C}$ are at high risk of bacteremia with S. pneumonia. Unexplained persistent and/or recurrent fever $\left(>37.5^{\circ} \mathrm{C}\right.$ intermittent or constant) for $>1$ month is considered as a moderately severe HIV (Clinical Stage 3, WHO HIV Criteria, August 2006).

In a study of 316 febrile children with advanced HIV disease [51], the diagnoses were

- disseminated mycobacteria avium complex (MAC) infection in 36\%;

- tuberculosis in $16 \%$;

- B lymphoma (6\%);

- disseminated CMV infection (4\%);

- extrapulmonary cryptococcosis $(3.5 \%)$;

- cases presented as PUO (17\%);

- other less frequent diagnoses such as drug fever, endocarditis, HIV primary infection, pancreatic abscess, Pseudomonas aeruginosa bacteremia, and visceral leishmaniasis.

Most of these patients (about 75\%) were receiving cotrimoxazole prophylaxis with or without antiretroviral treatment.

Children with HIV infection often present with the following:

1. Fever without focal signs and duration $<14$ days in HIV (see Table 5.10);

2. Fever without focal signs and duration $>14$ days (see Table 5.11).

Evaluation of fever among patients with HIV infection requires a detailed history, focusing on:

- duration of fever;

- recent travel to an area of malaria or dengue disease;

- skin rash, cough, pain during swallowing, headache, diarrhoea, dysuria;

- weight loss;

- the last CD4 cell count (?) (normal CD count: 600-1000); and

- current treatment (e.g., ART) and adherence.

Examination should include routine physical examination focusing on areas likely to be involved in the infection, such as thorough palpation of the lymphnodes, neurological examination, and fundoscopy for cytomegalovirus (CMV) and TB.

Laboratory investigations should include the following:

- Blood tests: Complete blood count with differential counts; blood chemistry (transaminases, alkaline phosphatase, LDH); blood smear for malaria; serum cryptococcal antigen test $=\mathrm{SCrAg}$ ); dipstick for malaria (rapid tests), if in endemic zone; viral load, CD4 count; dengue serology (if patient is living or have travelled to endemic areas);

- Urinalysis;

- Chest X-ray, abdominal ultrasound;

- Stool examination for bacterial culture and acid-fast bacilli (AFB);

- Lumbar puncture (Gram stain, Ziehl-Neelsen, of glucose/protein levels;

- Mantoux Test or INF- $\gamma$ assay. 
Table 5.10 Differential diagnosis of fever without focal signs and duration $<14$ days in HIV infected children

\begin{tabular}{|c|c|}
\hline Differential diagnosis & Diagnostic clues \\
\hline Malaria & $\begin{array}{l}\text { - Living or a history of visiting malaria area } \\
\text { - Blood film/dipstick positive } \\
\text { - The presence of anaemia, low platelets }\end{array}$ \\
\hline Typhoid fever & $\begin{array}{l}\text { - Seriously ill without apparent cause } \\
\text { - Abdominal tenderness } \\
\text { - Relative bradycardia in relation to body temperature } \\
\text { - Maculopapular rash, often sparing palms }\end{array}$ \\
\hline Urinary tract infection & $\begin{array}{l}\text { - Dysuria, frequency, pyuria, tenderness in renal angles } \\
\text { - Positive nitrate and WBC dipstick }\end{array}$ \\
\hline Dengue & $\begin{array}{l}\text { - Patient from areas at risk } \\
\text { - Sudden onset of high fever with headache, pain behind eyes, } \\
\text { joint and muscle pain. } \\
\text { - Macular rash in } 50 \% \text { (centrifugal, itching) (In dengue haemor- } \\
\text { rhagic fever, there is in addition bleeding tendency, eg from } \\
\text { the nose, bowel, fingers) }\end{array}$ \\
\hline Septicemia & - Seriously ill with no apparent cause \\
\hline $\begin{array}{l}\text { Immune reconstitution } \\
\text { Inflammatory syndrome }\end{array}$ & $\begin{array}{l}\text { - Recent start of HAART } \\
\text { - CD } 4 \%<10 \% \text { at start of HAART } \\
\text { - Rise in } \mathrm{CD} 4{ }^{+} \text {lymphocyte count }\end{array}$ \\
\hline Drug-induced fever & $\begin{array}{l}\text { - Nevirapine, cotrimoxazole, dapsone, } \beta \text {-lactams, isoniazid, } \\
\text { anticonvulsants, abacavir, efavirenz }\end{array}$ \\
\hline
\end{tabular}

HAART highly active antiretroviral treatment

Management of newly diagnosed children who present with PUO entails the following:

- A thorough search and adequate treatment of the secondary infections should be carried out prior to starting the antiretroviral therapy.

- The above investigations should be initiated prior to any treatment.

- Antibiotics to cover likely infections and particularly S. Pneumonia while waiting for results may be required.

- Patients with confirmed or probable TB (abnormal chest X-ray, positive gastric aspirate/ sputum AFB, abdominal lymphadenopathy, positive INF- $\gamma$ release assay) should start on antituberculosis treatment. This treatment is also indicated as an empirical therapy in cases of unexplained weight loss and fever in advanced AIDS.

- If a patient is not improving on anti-TB treatment, alternative diagnoses such as mycobacteria avium complex (MAC) should be considered.

- Patients responding to MAC therapy should continue until CD4 cells have adequately recovered. This may takes months/years.

- During the whole period of treatment, patients should repeatedly be re-evaluated for the appearance of new symptoms and signs, which may indicate additional infections.

Management of HIV patients with PUO while taking antiretroviral therapy should involve the following: 
Table 5.11 Differential diagnosis of fever without focal signs and duration $>14$ days in HIV infected children

\begin{tabular}{|c|c|}
\hline Differential diagnosis & Diagnostic clues \\
\hline Disseminated TB & $\begin{array}{l}\text { Advanced HIV/AIDS, with anemia } \\
\text { AFB seen on sputum, gastric aspirate, CSF, pleural fluid } \\
\text { and/or fine needle aspirate of lymph nodes } \\
\text { Enlarged mediastinal or hilar lymph nodes, pulmonary } \\
\text { infiltrates or miliary lesions on chest X-ray. Enlarged } \\
\text { liver or spleen, or enlarged lymph nodes on abdominal } \\
\text { ultrasound }\end{array}$ \\
\hline $\begin{array}{l}\text { Mycobacterium avium Complex } \\
\text { (MAC) }\end{array}$ & $\begin{array}{l}\text { Severe immunosuppression or WHO stage } 4 \text { disease } \\
\text { Symptoms compatible with disseminated TB, but failing } \\
\text { to respond to TB medicines } \\
\text { Absence of peripheral lymphadenopathy } \\
\text { Severe anemia and neutropenia }\end{array}$ \\
\hline Cytomegalovirus (CMV) & $\begin{array}{l}\text { Very low CD } 4 \text { count }\left(\mathrm{CD} 4<50 \text { cells } \mathrm{mm}^{-3} \text { in children }\right. \\
>5 \text { years) or WHO stage } 4 \text { disease. Blind spots in one or } \\
\text { both eyes, with signs of retinitis }\end{array}$ \\
\hline $\begin{array}{l}\text { Disseminated cryptococcal } \\
\text { infection }\end{array}$ & $\begin{array}{l}\text { Very low } \mathrm{CD} 4 \text { count }\left(\mathrm{CD} 4<50 \text { cells } \mathrm{mm}^{-3} \text { in children }\right. \\
>5 \text { years) or WHO stage } 4 \text { disease } \\
\text { Headache, Molluscum-like skin lesions } \\
\text { Positive serum cryptococcal antigen tests } \\
\text { Isolation of the pathogen from CSF, lymph nodes, spu- } \\
\text { tum, or skin ulcers (indian ink). }\end{array}$ \\
\hline Visceral leishmaniasis & $\begin{array}{l}\text { Splenomegaly, lymphadenopathy, pancytopenia } \\
\text { Amastigotes seen in samples of tissue or body fluid under } \\
\text { the microscope (Giemsa stain) }\end{array}$ \\
\hline Bacterial endocarditis & $\begin{array}{l}\text { Enlarged spleen, heart murmur petechiae on skin and } \\
\text { mucosa, anaemia. } \\
\text { Splinter hemorrhages in nail bed }\end{array}$ \\
\hline Relapsing fever & $\begin{array}{l}\text { Exposure to ticks or body lice } \\
\text { Recurrent pattern of fever. Headache, muscle pain, } \\
\text { enlarged liver and spleen, red eyes, and photophobia }\end{array}$ \\
\hline Abscesses & Tender or fluctuant mass, often detected by ultrasound \\
\hline Trypanosomiasis & $\begin{array}{l}\text { Travel to or living in region with tsetse flies } \\
\text { History of painful trypanosomial chancre at site } \\
\text { of inoculation of the parasite. } \\
\text { Bouts of high fever lasting several days separated by } \\
\text { afebrile periods, lymphadenopathy, itching, and } \\
\text { maculopapular rashes }\end{array}$ \\
\hline
\end{tabular}

$A F B$ acid-fast bacilii; $C S F$ cerebrospinal fluid; $W H O$ World Health Organization.

- An associated skin rash should arouse the suspicion of drug fever. Nevirapine is a frequent cause of this. A common finding is elevation of liver transaminases. Abacavir is also a cause of hypersensitivity reactions and should never be used again, as it can be fatal.

- Patients who initially responded to treatment for opportunistic infections (OI) prior to the start of antiretroviral therapy, and then developed a worsening of the OI after the start of antiretroviral therapy (e.g. reappearing of fever), should be considered as having 
IRS (if other obvious causes of fever are excluded). Patients should continue OI therapy and ART but steroids treatment should be considered.

\section{8 \\ Infection of the CNS}

\subsection{1}

\section{Meningitis}

Meningitis remains one of the most important infectious causes of neurodisability and death in childhood. Newborn infants and children between 6 and 12 months of age are at greater risk of meningitis than older children; $90 \%$ of reported cases occur below 5 years of age. Congenital and acquired T- and B-cell defects, sickle-cell anemia, splenectomy, and malnutrition all predispose to meningitis. Definitions of the clinical variations of central nervous system (CNS) infection are provided in the Table 5.12.

The widespread use of vaccines against Neisseria meningitides and $H$. influenzae type B (Hib) has virtually eradicated the incidence of these forms of meningitis and their complications in well-immunized populations. The incidence of bacterial meningitis in the United States has decreased by 55\% since the introduction of Hib vaccines in 1990 [52]. This has lead to an increase of the median age of patients with bacterial meningitis to 39 years. In developing world with low immunization rates, however, these types of bacterial meningitis still occur. The commonly used antibiotic prophylaxis of pregnant women for Group B streptococci and the recent introduction of vaccines against pneumococcal infection have the potential to reduce the incidence of this disease even further.

Meningitis occurs most commonly in the individual who bears the organisms as an asymptomatic carrier. Organisms enter the CNS through vulnerable sites in the bloodbrain barrier (choroid plexus or cerebral microvasculature). The cell wall components of these organisms stimulate macrophage-equivalent brain cells (astrocytes, microglia). Once

Table 5.12 Definitions of meningitis, meningococcal disease, and encephalitis

- Confirmed meningitis: isolation of bacteria from CSF, blood or DNA detection through PCR from a patient with a CSF white cell count $>10$ cells $\mathrm{mm}^{-3}$. Diagnosis is also accepted in case of postmortem diagnosis of meningitis or meningeal contrast enhancement on CT scan

- Probable meningitis: the presence of clinical symptoms and signs of bacterial meningitis in the absence of laboratory confirmation

- Meningococcal disease: a clinical condition caused by Neisseria meningitidis with purulent conjunctivitis, septic arthritis, and septicemia with or without meningitis

- Aseptic meningitis: the presence of CSF white cell count $>10$ cells $\mathrm{mm}^{-3}$; CSF is negative for bacterial culture, occurring usually in summer months. Viruses are most common causes

- Encephalitis: an inflammation of the parenchymal tissue of the brain caused by an infection producing varying degrees of impaired consciousness

CSF cerebrospinal fluid; $P C R$ polymerase chain reaction; $C T$ computerized tomography. 
bacteria reach the CSF, they are likely to survive because humural defenses, including immunoglobulin and complement and opsonic activities, are virtually absent. Meningitis may also result from hematogenous dissemination, or rarely by direct invasion from ear or sinus infection. Data suggest that several cytokines, particularly IL-1 $\beta$, TNF- $\alpha$, and IL-6, are increased in children with meningitis (Fig. 5.2).

\subsection{2}

\section{Bacterial Meningitis}

Neonatal meningitis is most common during the first week of life (early onset; beyond the first week of life it is termed late onset). The susceptibility of neonates to meningitis, particularly premature infants, is mainly due to immaturity of cell- and antibody-mediated immune mechanisms. The neonate is infected by bacteria from the maternal genital tract, the risk being higher after membrane rupture.

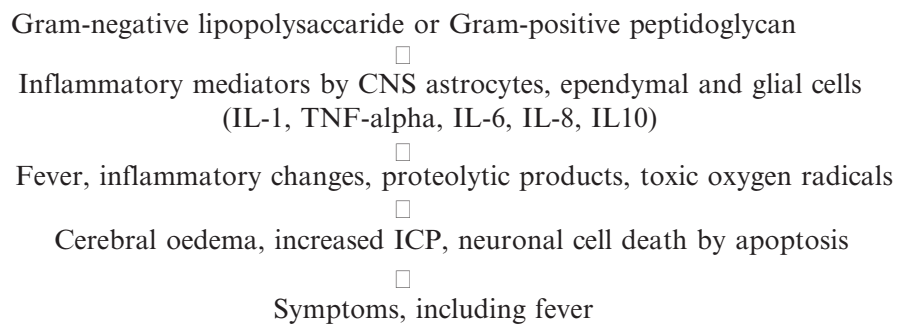

Fig. 5.2 Pathogenesis of meningitis and fever

Table 5.13 Causes of bacterial meningitis

\begin{tabular}{ll}
\hline Neonate & \\
$\bullet$ & (Caused by vertical transmission) \\
& Group B streptococcus (GBS) \\
& Escherichia coli $($ E. coli) \\
& Listeria monocytogenes \\
& Coagulase-negative staphylococci \\
& H. influenzae \\
& (caused by nosocomial or community spread) \\
& Gram negative enteric bacteria (GNEB) \\
& (E.coli, klebsiella, enterobacter, salmonella, proteus, pseudomonas) \\
& In developing countries: GNEB, H. influenzae \\
& \\
& Neisseria meningitidis \\
& Streptococus pneumoniae \\
& H. influenzae \\
\hline
\end{tabular}


A study of 274 neonates from England and Wales established an annual incidence of bacterial meningitis at 0.21 per 1000 births [53]. The overall case fatality rate was $6.6 \%$. Group B streptococcus remains the leading pathogen (about $50 \%$ of cases, Table 5.13). In contrast to older children, the onset of neonatal meningitis is usually insidious. Infants present with the following:

- Symptoms such as failure to feed, lethargy alternating with irritability, seizures, vomiting, thermal instability (fever or hypothermia), cyanosis, apnea, jaundice; and respiratory distress;

- Signs such as an ill appearance, a tense or bulging fontanelle, pallor, and reduced capillary refill time. Neck stiffness and head retraction are not parts of the symptomatology.

Complications include hydrocephalus, ventriculitis, neurodisability, and seizures.

Meningitis in older children is mostly meningococcal (in combination with sepsis called meningococcal disease, $\mathrm{MCD}$ ) or pneumococcal. Less common causes are E. coli, group B streptococci, staphylococci, Listeria, Borrelia burgdorferi (Lyme disease), TB, and fungi. Factors that increase the risk for bacterial meningitis include immunoglobulin deficiency (e.g., HIV infection), asplenia, neuro-surgical procedures (e.g., ventriculoperitoneal shunt), penetrating head injury, and cochlear implants (particularly for pneumococcal meningitis).

Meningitis has a variety of presentations:

- In MCD, the nonspecific early symptoms (in the first 4-6h) are fever, irritability, and decreased appetite. This is followed (at a median time of $8 \mathrm{~h}$ ) by early symptoms of sepsis: leg pain, abnormal skin color, and cold hands and feet. Classic meningitis symptoms appear later (13-22 h): purpuric rash, impaired consciousness and meningism [54];

- Fever, vomiting, irritability or drowsiness, headache, and photophobia;

- Convulsive status epilepticus with fever.

Tuberculous meningitis compromises about $5-10 \%$ of extrapulmonary cases of TB. It often occurs within 6 months of the initial TB infection following hematogenous dissemination or a rupture of a subependymal focus into subarachnoid space. The incidence is highest in children aged 1-5 years. The three recognized stages are:

- conscious, with nonspecific symptoms (fever, night sweats, anorexia, weight loss, fatigue) and no neurological signs;

- onset of neurological signs: headache, confusion, drowsiness, neck stiffness; and

- stupor, deepening coma, focal neurological signs.

Fever in bacterial meningitis is:

- the most common presenting symptom in children beyond the neonatal age owing to the presence of inflammatory mediators, particularly IL-1 and TNF in blood or within the CNS. In MCD, fever was the first symptom in children younger than 5 years, and $94 \%$ developed fever at some point;

- uncommon in neonatal meningitis. It occurred in about $30 \%$ of 36 neonates with $E$. coli meningitis [55]. Neonates have a reduced capacity to produce cytokines, which may explain their frequent afebrile presentation; 
- usually very high in older children. Temperatures between 40 and $41^{\circ} \mathrm{C}$ are common, with a mean degree of $39.2^{\circ} \mathrm{C}$. The degree of fever varies depending on the age of the patient and the causative organisms. The incidence of fever was $71 \%$ with meningococcal infection, $88 \%$ with staphylococcal infection, and $90 \%$ with $\mathrm{H}$. influenza type B. Children with TB meningitis have the highest incidence of fever with 97\% [56];

- absent in rare cases of severe infection. Hypothermia carries a bad prognosis;

- short and often settles within 24-48h in MCD. Its duration in pneumococcal infection is significantly longer than in other types of bacterial meningitis.

Fever is an important sign when monitoring the effect of treatment in bacterial meningitis; that is, normalization of fever is very suggestive of a good response and improvement. Non-responders may produce the following fever patterns:

- Persistent for 4-7 days

- Prolonged, more than 7 days

- Secondary fever (fever reappearing after at least one afebrile day).

The following considerations should be made in persistent or prolonged fever:

- The antibiotics or the doses used for meningitis therapy are inappropriate (e.g., penicillin administered for staphylococcal meningitis);

- The meningitis may be aseptic or TB meningitis;

- There are complications listed in Table 5.14;

- The child needs thorough re-evaluation to find out the cause of the fever;

- Morbidity and mortality are higher than in those cases who have responded to treatment.

Animal models of meningitis have provided substantial information on the pathophysiology of fever in the disease. Studies [57,58] investigating the influence of fever on experimental meningitis in rabbits concluded that high body temperature had a direct inhibiting

Table 5.14 Causes of persistent, prolonged, and secondary fevers

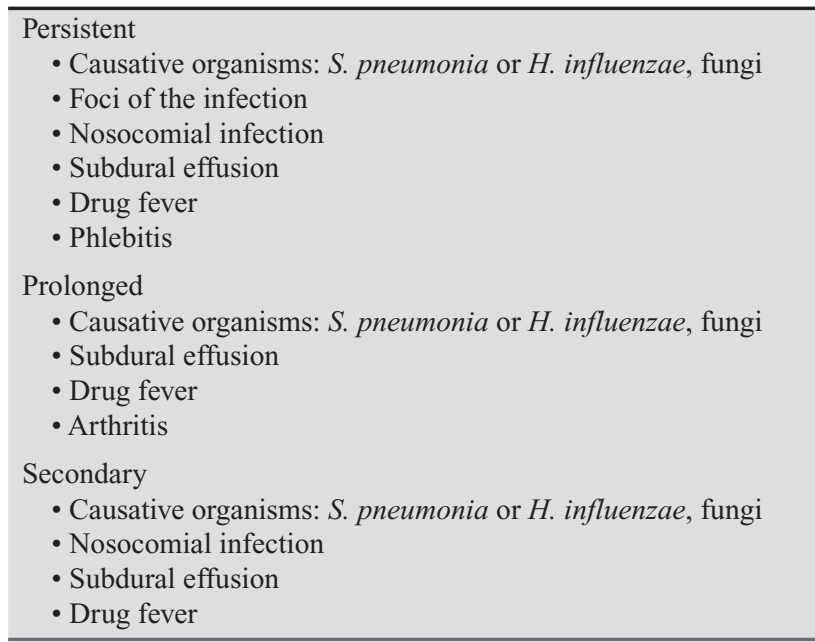


effect on the growth rate of bacteria in the CSF. On the other hand, the lower the temperature, the faster was the rate of bacterial growth. Thus fever is likely to be a host defense in this disease. Similar results are available in human studies. The reported overall case fatality rate in 100 children with meningococcal infection ( 55 had meningitis) did not indicate a poor prognosis, but all children with hypothermia died [56].

In a study of 476 children with meningitis, $90 \%$ of patients with S. pneumoniae and N. meningitidis became afebrile within five days of the antibiotic therapy, compared to $72 \%$ of those with $\mathrm{H}$. influenzae meningitis. The rate of persistent fever, prolonged fever, and secondary fever was 13,13 , and $16 \%$, respectively [59]. Complications from meningitis including persistent and prolonged fever, have decreased following decreased incidence of meningitis due to routine H. influenza vaccination in 1992 and recently Pneumococcal vaccine, but still occur with other types of bacterial meningitis.

Laboratory findings include the following:

- Characteristic CSF findings (Table 5.15) and identification of the pathogens in CSF and/or blood cultures and/or PCR. In TB meningitis, additional positive findings are microscopy for AFB from CSF, gastric aspirate, sputum (ZN stain), tuberculin test $>5$ or 10 induration, history of TB contact, and radiological evidence in the CNS of tuberculoma and/or other changes, such as hydrocephalus;

- A polymorphonuclear leukocytosis and high CRP in the blood;

- High procalcitonin (PCT) level $\left(>0.5 \mathrm{ng} \mathrm{m}^{-1}\right)$. PCT is a precursor of calcitonin, which may be more valuable than CRP and IL-6 in differentiating bacterial from viral meningitis early. PCT is also higher in severe compared to mild disease.

- Other abnormalities are inappropriate secretion of antidiuretic hormone (ADH) with hyponatremia, water retention increased intracranial pressure and DIC. DIC manifests as thrombocytopenia, increased fibrin degradation products, and prolonged prothrombin (PT) and partial thromboplastin time (PTT).

Complications include seizures, neurodisability, paralysis of the cranial nerves, subdural collection, blindness, hydrocephalus, cerebral herniation, and deafness.

Table 5.15 Usual cerebrospinal fluid findings in normal and in various central nervous system, (CNS) infections

\begin{tabular}{llll} 
Normal & Cell mm $^{-3}$ & $\begin{array}{l}\text { Protein } \\
\text { mg dl }^{-1}\end{array}$ & $\begin{array}{l}\text { CSF/serum } \\
\text { glucose ratio \% }\end{array}$ \\
\hline Normal & $0-5$ (lymphocytes) & $20-40$ & $>50$ \\
Bacterial meningitis & $100-1000($ PMN) & $100-500$ & $<0.5$ \\
Tuberculosis & $30-600($ lymphocytes) & $>100$ up to $3 \mathrm{~g}$ & $<0.5$ \\
Viral meningitis & $100-2000$ (lymphocytes) $^{\mathrm{a}}$ & Normal to 200 & Normal \\
Encephalitis & Normal to few hundreds & $50-100$ & Normal \\
Abscess & $10-100($ PMN per & $30-200$ & Normal \\
& lymphocytes) & & \\
\hline
\end{tabular}

$P M N$ polymorphonuclear cells

a PMN predominate initially with lymphocytes predominating after $48 \mathrm{~h}$ 
Table 5.16 Treatment recommended for children with tuberculosis

\begin{tabular}{|c|c|c|c|}
\hline \multirow[t]{2}{*}{ Drugs } & \multirow{2}{*}{$\frac{\text { Daily dose }}{\mathrm{Mg}}$} & Maximal daily & \multirow[t]{2}{*}{ Major side effects } \\
\hline & & $\mathrm{mg} \mathrm{kg}^{-1}$ per day & \\
\hline $\mathrm{INH}$ & 10 & 300 & Peripheral neuropathy \\
\hline Rifampicin & $10-20$ & 600 & Hepatitis \\
\hline Pyrazinamide & $25-35$ & $2 \mathrm{~g}$ & Hepatotoxicity \\
\hline Ethambutol & $15-20$ & $2 \mathrm{~g}$ & Optic neuritis \\
\hline Streptomycin & 40 & $1 \mathrm{~g}$ & Ototoxicity \\
\hline
\end{tabular}

Therapy consists of prompt i.v. administration of antibiotics. Neonates are treated with cefotaxime, penicillin (or ampicillin), and gentamicin for a duration of 2 (GBS and Listeria) or 3 weeks (Gram-negative bacteria). Older children are treated with third-generation cephalosporin cefotaxime or ceftriaxone. Treatment of TB meningitis is shown in Table 5.16.

Dexamethasone has been advocated for the treatment of bacterial meningitis. Early administration of dexamethasone has been shown to reduce the duration of fever, levels of cytokine concentration, and the incidence of hearing impairment. It is mainly beneficial for $H$. influenzae and S. pneumoniae meningitis if it is given with or before antibiotics.

Intravenous fluid should be restricted to minimize the effect of inappropriate ADH effect and the cerebral edema. Monitoring the electrolytes and body weight are important for the management of the fluid and electrolyte balance.

The presence of coma, shock, seizures, and hypothermia are associated with poor prognosis. Children with TB meningitis usually make full recovery if they are fully conscious at presentation, while those in coma have a high rate of neurodisability and deaths. The younger the child, the worse the prognosis.

\section{Viral Meningitis}

The true incidence of viral meningitis is unknown mainly because CSF with aseptic meningitis is often not examined for viruses. The incidence of proven viral meningitis is 0.05 per 1000 live births [53]. The most frequent etiological agents remain non-polio enteroviruses (echovirus and coxsackievirus). Mumps meningitis, which used to be the most common form of viral meningitis prior to the combined measles, mumps, and rubella (MMR) vaccination in 1988, has declined dramatically. Symptoms are similar to those of bacterial meningitis but they are usually mild and the children appear generally well. This infection affects mainly older children.

Fever varies usually between 38.5 and $39.5^{\circ} \mathrm{C}$, rarely higher. Fever along with drowsiness and irritability are the major presenting symptoms. The incidence of fever is around $70 \%$.

Of the various cytokines capable of inducing fever, INF- $\gamma$ produced in the intrathecal space appears to be associated with the pathogenesis of viral meningitis, and the production of fever. CSF INF- $\gamma$ levels correlate well with the severity of febrile episodes.

Laboratory findings include clear, or rarely opalescent, CSF (Table 5.15). CRP and WBC are usually normal. Procalcitonin is a useful marker to differentiate bacterial and aseptic meningitis.

Diagnosis requires isolation of the specific virus from the CSF and/or a fourfold rise in antibody titer to the virus. Rapid identification of the virus by immunofluroscent examination of the CSF is possible for many viruses. The prognosis is very good. 


\subsection{3}

\section{Acute Viral Encephalitis}

This is an illness with an acute onset and rapid progression caused commonly by the herpes simplex virus (HSV). Other viruses include varicella, cytomegalovirus, EBV, coxackievirus, echovirus, poliovirus, mumps, measles and adenovirus. The annual incidence is 8.8 per 100,000 children younger than 16 years of age [60].

Clinical features vary depending on the nature of the causative virus, the age of the patient, and the severity of the infection. Commonly, the disease begins with an acute onset of fever, headache, and vomiting. Evidence of meningeal irritation and stiff neck is often lacking. Encephalitis is suggested by drowsiness, paralysis, coma, seizure (febrile seizure), ataxia, tremor, mental confusion, or hyperexcitability. Ataxia is common, particularly following varicella encephalitis.

Fever is common in viral encephalitis irrespective of the causative agent. It was present in $30 \%$ patients with mumps encephalitis, in $85 \%$ with coxsackievirus B encephalitis, and in $90 \%$ of patients (a third had a fever greater than $39^{\circ} \mathrm{C}$ ) with herpes encephalitis [61]. Fever, lethargy, and headaches may last 4-5 days before other symptoms (such as behavioral abnormalities) occur.

Laboratory diagnosis of herpes encephalitis mainly depends on PCR detection from the CSF, which is highly sensitive and specific. Electroencephalogram (EEG) commonly shows paroxysmal focal abnormalities (such as slow complexes every 2-3s) over the involved temporal areas. A computerized tomography (CT) scan of the head may show characteristic low-density lesions in these areas, in addition to diffuse brain edema. Magnetic resonance imaging (MRI) is a superior investigation for showing lesions in the temporal areas, uni- or bilateral.

Therapy with acyclovir should be initiated to all cases with suspected encephalitis while awaiting laboratory confirmation.

Subacute sclerosing panencephalitis (SSPE) is a progressive inflammatory disease of the CNS caused by persistent, aberrant measles virus infection, characterized by progressive loss of intellectual function, with behavior and learning difficulty, often associated with abnormal myoclonic movements. High anti-measles antibody titers in serum and CSF confirm the diagnosis. The mean interval between measles and the onset of SSPE is about 10 years. The MMR vaccine has resulted in virtual elimination of SSPE. Fever is not part of SSPE.

\subsection{4}

\section{Brain Abscess}

Brain abscess is uncommon in children. It may occur as a complication of $\mathrm{OM}$, mastoiditis, sinusitis or meningitis, or ventriculoperitoneal shunt infection, following trauma or surgery to the skull, or as a result of haematogenous dissemination in children with acyanotic congenital heart disease.

Fever was the most common clinical finding in a study [62] of 101 children with brain abscess, occurring in $80 \%$ of the children, followed by vomiting, headache, seizure, focal neurological abnormalities, and lethargy. Papilledema and meningeal signs were also common. Overall mortality was $30 \%$. High fever, age less than 1 year, multiple brain foci, and the presence of meningism or coma have a poorer prognosis. 
The most frequently encountered pathogens are S. aureus, streptococci and Gramnegative aerobic bacilli.

Laboratory findings in the CSF reveal that the CSF culture is usually negative unless there is rupture of the abscess into ventricles. A CT scan shows the characteristic finding of a ring-enhancing lesion.

Therapy consists of antimicrobial treatment (third-generation cephalosporin, vancomycin, and metronidazole) with or without surgical excision or aspiration.

\section{9 \\ Osteomyelitis and Septic Arthritis}

(See also Chapter 12: Differential Diagnosis: Arthritis)

Infection of the bone may occur as a complication of septicemia or due to local trauma (e.g., wound, abrasion). Acute hematogenous osteomyelitis involves most commonly the rapidly growing metaphysis of the long bones. The femur and tibia are most commonly affected bones. Septic arthritis is usually hematogenously acquired or the result of an extension from an osteomyelitic lesion. The knee is most commonly involved. S. aureus is the most frequent bacteria causing the infection (accounts for $90 \%$ ), followed by kingella kingae, $S$. pneumonia, $S$. pyogenes, and $P$. aeruginosa, which are less common causes. Children with sickle-cell anaemia and other hemoglobulinopathy are at high risk of osteomyelitis caused by non-typhi salmonella.

This infection presents:

- In neonates with irritability and tenderness when the affected area is touched and limited movement of the affected extremities (pseudoparalyis). Fever is either mild or absent;

- In older children with high fever, refusal to walk, bone pain, and limping (if the lower extremities are affected). Examination reveals localized pain, tenderness, warmth, and erythema of the affected area.

The diagnosis is based on the following criteria:

- The isolation of bacterial pathogens or positive PCR from blood (positive in 30-60\%), bone, or joint. Needle aspiration of the soft tissue or incision and drainage of the bone may yield the organism. In septic arthritis, joint fluid aspiration usually reveal purulent exudates with $>50,000$ leukocytes $\mathrm{mm}^{-3}$, Gram-positive cocci, and a positive culture. Leukocytosis and elevated CRP are usually present. CRP is a very reliable parameter to assess the effectiveness of the treatment and recovery.

- Radiological findings (soft-tissue swelling, bone rarefaction, periosteal elevation, bone necrosis) may not appear during the first two weeks of the infection. A nuclear bone scan (showing increased uptake of the isotope) is a valuable adjunct to the diagnosis and is often positive before the appearance of the lesion in the X-ray.

Fever is the most common presenting symptom of bone infection, occurring in $90 \%$ of admission with a mean temperature of $39.1^{\circ} \mathrm{C}$ [63]. Most of those who were afebrile on admission became febrile during the ensuing $48 \mathrm{~h}$ after admission. Normalization of fever is not usually achieved during the first week despite antibiotic treatment. High fever usually 
continues for 4-5 days after the treatment. Therefore the presence of persistent and high fever during treatment does not necessarily signify failure of antibiotic treatment.

Initial antibiotics are likely to include i.v. ceftriaxone with clindamycin, flucloxacillin, or Fucidin for 3-6 weeks.

\subsection{0 \\ Viral Exanthems}

Viral exanthems are common causes of febrile illness in children. More than 50 viral agents are known to cause a rash. Historically, exanthems were numbered in the order in which they were differentiated from other exanthems. Thus the first was measles; second scarlet fever; third rubella; forth the so-called Filatov-Dukes disease (no longer recognized as an entity); fifth erythema infectiosum, sixth exanthema subitum. As more exanthems were described, numerical assignment became impractical.

\subsection{0 .1}

Measles

The first written record of measles is credited to Rhazes, a Persian physician of the tenth century; before that measles was thought to be a mild form of smallpox. Sydenham in the seventeenth century drew an accurate clinical picture of the disease, including recognition of its complications. When the United States was swept by measles during the seventeenth and eighteenth centuries, the infection was still believed to be a sequel to smallpox. Measles virus was cultivated in 1938 .

Prior to the present vaccine, the attack rate of measles worldwide was close to $100 \%$ and measles was an important cause of mortality. In the United States, in 1949, measles ranked seventh among the causes of death for ages 1-4 years, which were mainly due to pneumonia. Following routine vaccination in 1963, measles has been almost eliminated. In the United Kingdom, the mortality rate until 1950 was 1 per 1000 measles cases. Following routine vaccination in 1968, the mortality rate decreased to 1 per 5000 [64].

In developing countries without immunization, measles affects virtually all children by the age of 4 years, the highest incidence being in the second half of the second year. Mortality in the past has ranged between 15 and $25 \%$. More recently, this mortality has decreased progressively in developing countries, for example, in India and Africa. The single most important factor affecting mortality is poor nutritional status, leading to deficiencies in cell-mediated immunity and often death due to giant-cell pneumonia, diarrhea, or inclusion body encephalitis.

Measles is caused by paramyxovirus, which spreads by droplets from person to person. The incubation period is about 11 days. The spreads of the virus occurs through the following steps:

- Virus enters the epithelium of the conjunctiva and upper respiratory tract;

- Viral antigens presenting cells of the mononuclear phagocytic system, followed by expansion of antigen-specific T lymphocytes; 
- High affinity IL-2 receptors rise before the onset of the rash and remain elevated for several weeks;

- Following its spread by day 5 to the mononuclear phagocytes of the liver and spleen, the virus continues its spread by day 8 via the blood to its target tissue (eye, lung, and gut epithelial cells). During these stages, viral spread is limited by natural killer cells and cytotoxic T cells. B cells are primed to produce antibody.

Clinically, the infection progresses through the following steps:

- The pre-exanthem stage expresses like a common cold, with abrupt fever, sneezing, dry cough, and conjunctivitis. The temperature increases gradually to reach a level ranging from 39 to $40.5^{\circ} \mathrm{C}$. About $24 \mathrm{~h}$ prior to the appearance of exanthema, Koplik's spots can be detected in about $80 \%$ of cases as tiny (about $1 \mathrm{~mm}$ ) whitish spots in the buccal mucosa opposite the lower morals.

- The exanthem appears at the peak of symptoms with a temperature of about $39.5^{\circ} \mathrm{C}$. The rash appears first behind the ears and spreads to the face, neck, trunk, and extremities. The rash begins to clear on the third day. During the exanthem period, the fever usually peaks on the second or third day, and then falls by lysis over a 24-h period. Fever that persists after the third day may signify bacterial complication. There are signs of pharyngitis, cervical lymphadenopathy, and occasionally a mild splenomegaly. Shortly after the rash appears, the child becomes anergic, with suppression of the delayed hypersensitivity to skin test antigens and reduced lymphoproliferation and lymphokine production in response to mitogenic stimuli. The infectivity decreases considerably with the onset of the rash.

Blood counts often show leucopenia and lymphopenia. Suppression of immune function is manifested in vivo by the loss of response to tuberculin skin test. On the other hand, there is an increased activation of lymphocytes and macrophages, plasma INF- $\gamma$ and IL-2 receptors. The diagnosis of measles can be confirmed by measles complement fixation or hemagglutination antibody test.

Complications include pneumonia (viral or bacterial), OM, gastroenteritis, laryngitis, encephalitis, bronchiectasis, reactivation of tuberculosis, and SSPE.

Gamma globulin $\left(0.25 \mathrm{mg} \mathrm{kg}^{-1}\right)$ within 5 days of exposure to measles virus prevents the disease. Oral vitamin A $(400,000 \mathrm{U})$ can decrease mortality in children in developing countries.

\subsection{0 .2 Varicella}

Varicella zoster virus is a member of the herpesvirus family. The eruption is often the first sign of the onset of varicella, particularly in young children. Older children and adults may have prodromal symptoms preceding the characteristic eruption by 1-2 days, which include fever in the range of $38-38.5^{\circ} \mathrm{C}$ (temperature up to $40.5^{\circ} \mathrm{C}$ may occur), malaise, headache, and abdominal pain. The characteristic eruption of macules and papules appears first on the back, then on the rest of the trunk, spreading within hours to the face and scalp. The lesions progress from macules to papules to vesicles and begin crusting within 8-10h. Characteristically, these lesions are found simultaneously. The highest body temperature of up to $40.5^{\circ} \mathrm{C}$ occurs during the first 3 days of eruption and falls to a normal level on the fourth day. 
When maternal varicella develops within 4 days of delivery, neonates develop severe varicella within 5-10 days postpartum. The disease is associated with a mortality of around $20 \%$ due to disseminated chickenpox, usually with severe pneumonitis. When maternal varicella develops 10-20 days before delivery, transfer of maternal antibodies causes a more benign illness.

Varicella is usually a benign disease. Complications include pneumonia in about $1 \%$ (affecting primarily adults and newborns), with a chest X-ray showing nodular infiltrates, secondary staphylococcal skin infection, thrombocytopenic purpura, cerebellar ataxia, and encephalitis. Secondary bacterial infection from Staphylococci and Streptococci can be fatal and will need urgent treatment. Varicella is severe and may be fatal in patients with impaired cellular immunity, such as those receiving cytotoxic drugs. Children with hypogammaglobulinemia recover normally from varicella.

Most patients require no special treatment. Aspirin should not be administered because of the risk of Reye's syndrome. Itching can be relieved by simple soothing lotions such as calamine and oral antihistamine. Patients with severe varicella or with complication should receive acyclovir. This antiviral drug promotes the cutaneous healing and reduces the duration of fever. A vaccine is now available and is increasingly being used to prevent this disease.

\subsection{0 .3}

\section{Rubella}

Rubella virus may cause inapparent or severe infection. The infection is usually mild, and children usually present with sore throat, rash, lymphadenopathy (prominent in the posterior cervical area), and low-grade fever (rarely exceeding $39^{\circ} \mathrm{C}$ ) for several days. Fever may persist for 1-2 days, rarely 3 days. In older children, particularly in females after puberty, the infection is more severe and prolonged. There are usually painful, with visibly enlarged lymph nodes involving postauricular, occipital, and posterior cervical nodes, with polyarthralgia or arthritis.

The infection with rubella virus is particularly important to pediatricians because of possible fetal-maternal transmission. Congenital infection (rubella syndrome) is highest in the early weeks of pregnancy, manifesting as eye disease (cataract, retinopathy, glaucoma), sensorineural deafness, heart lesions (patent ductus arteriosus, pulmonary artery stenosis, aortic stenosis, coarctation of the aorta or ventricular septal defect), neurological abnormalities, or thrombocytopenic purpura. Prevention of maternal rubella used to be through routine immunization of all girls of 11-14 years of age and women of child-bearing age, but the use of the MMR has been more successful in reducing rubella syndrome by preventing transmission of the virus from children to pregnant mothers.

\subsection{0 .4 \\ Erythema Infectiosum}

Erythema infectiosum (EI) or the fifth disease is an acute, benign communicable disease with a characteristic eruption. It usually affects children aged 5-15 years. The infection is caused by parvovirus B19, which also can cause a transient aplastic crisis in patients with 
hemolytic anemia and hemoglobulinopathy, and an arthritis similar to rheumatoid arthritis. The mean incubation time is 9 days (range 4-14 days).

The eruption is generally the first and only diagnostic clinical manifestation of the disease, occurring in $100 \%$ of cases. It starts on the face with a "slapped cheeks" appearance, resembling scarlet fever. The rash spreads to the trunk and extremities in 1-4 days after the onset of the facial rash. The rash is erythematous maculopapular and tends to assume a reticular or lacy pattern, which last for 4-6 days. Common associated clinical findings are pruritis, arthralgia/or arthritis (mainly in adults), and headache. Fever is observed in about $23 \%$ of cases. Encephalitis is a very rare complication.

\subsection{0 .5}

\section{Exanthema Subitum}

Exanthema subitum (ES) is caused by human herpes virus 6 (HHV-6), and was identified in 1988. The virus is recognized as a major of febrile illness with viremia and a high temperature (mean $39.7^{\circ} \mathrm{C}$ ), sometimes with rash. Occasionally the virus can cause an inapparent infection without fever, a rash without fever, or a fulminant hepatitis and death.

ES is the most common febrile exanthem in children under the age of 3 years. Approximately $30 \%$ of children develop this disease eventually. Ninety percent of all cases occur in children aged 6-24 months. The incubation period is between 5 and 15 days.

Before the onset, children may have a short period of irritability and malaise. Onset of fever is abrupt (sometimes triggering a febrile seizure) and characteristically continuous (or less commonly intermittent), often as high as $40-41^{\circ} \mathrm{C}$. The fever persists for $3-4$ days in about $75 \%$ and for $5-6$ days in the remaining $25 \%$. There is usually no focus to explain the presence of fever except often a mild pharyngitis, suboccipital or posterior cervical lymphadenopathy. The temperature usually drops by crisis over a period of a few hours, coinciding with the appearance of the rash (Fig. 5.3). The rash appears predominately on the neck and trunk, lasting 24-36h. Characteristically, the child becomes well and afebrile when the rash erupts.

When fever is intermittent, the temperature is normal or slightly elevated in the morning, only to rise to $40-40.5$ by early evening. Fever may fall by lysis over a period of $24-36 \mathrm{~h}$.

Laboratory findings commonly show leukocytosis of 12,000-20,000 with a slight increase in neutrophils.

\subsection{1 \\ Tropical Diseases}

\subsection{1 .1 \\ Tuberculosis}

Tuberculosis is a major cause of morbidity and mortality throughout the world. Although reported cases have declined, particularly in developed countries, 1-3 million still die annually from the disease. The number of TB cases in the United States has declined from 6036 in 1962 to 1261 in 1985; 80\% of them occurred in the minority groups living there 


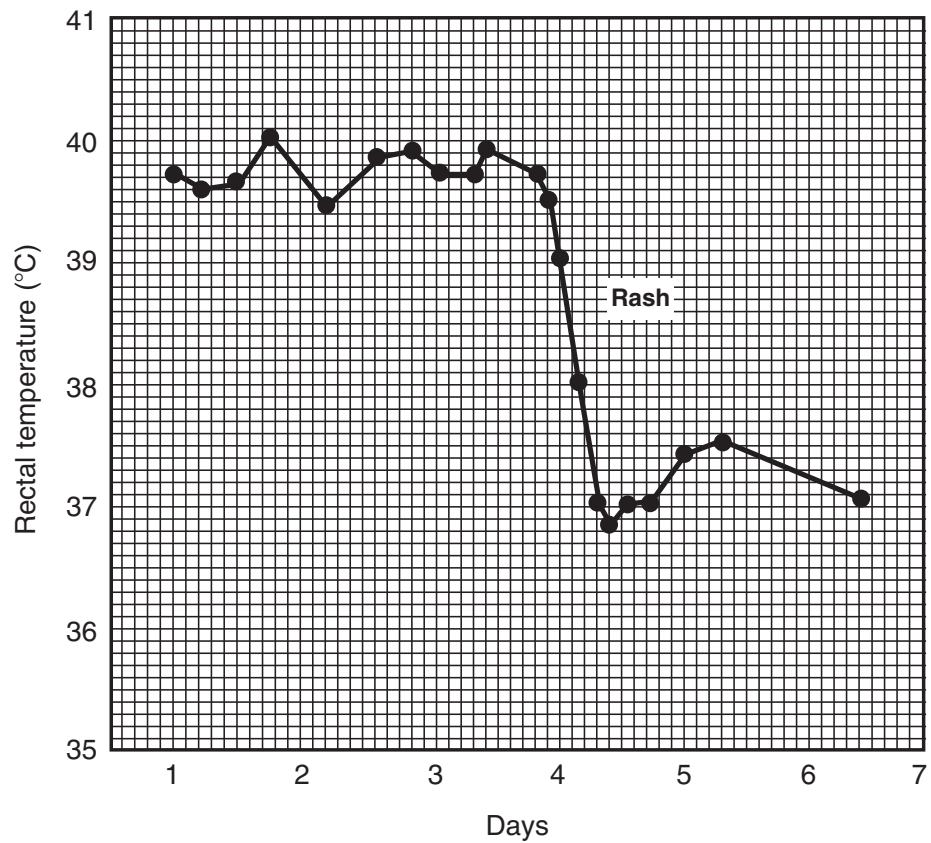

Fig. 5.3 Continuous fever pattern seen in erythema subitum, with a drop in temperature by crisis

[65]. However, after four decades of steady decline, the annual case rate leveled off in 1985 and has increased worldwide during the past two decades. Children acquire the infection from adults who have active disease and are expectorating tubercle bacilli. Children themselves are noncontagious. Therefore, every effort should be made to identify the adult source for eradicating the source.

When TB bacilli are inhaled, only the fine particles containing one to three tubercle bacilli reach the alveolar macrophages of the lung. Although macrophages are able to eliminate tubercle bacilli, they must be activated by T lymphocytes, especially INF- $\gamma$, in order to do so.

Neonatal TB occurs through transmission of infection from mother to infant via the placenta or amniotic fluid. Neonates present with feeding difficulty, failure to thrive, jaundice, respiratory distress, or hepatosplenomegaly. Fever is usually absent. Chest X-ray shows bronchopneumonia. The disease often runs a fulminant course with rapid multiplication of tubercle bacilli and minimal giant-cell formation.

In older children, primary infection (refers to infection in a person with no prior immunity) is asymptomatic in most cases. Occasionally there is low-grade fever $\left(38-38.5^{\circ} \mathrm{C}\right)$. Radiologically, a parenchymal lesion is usually not visible, but hilar adenitis is prominent and may cause compression of the adjacent soft bronchus, causing wheezing and nonproductive cough. With increased compression, or following perforation of an infected lymphnode into the bronchus, segmental atelectasis may ensue. Other presentations are erythema nodosum, phlyctenular conjunctivitis (as a result of hypersensitivity reaction), 
or TB pneumonia, which resembles radiologically bacterial pneumonia with high fever, cough, and dyspnea.

Miliary TB in children usually presents with no specific symptoms and signs. Fever is present in about $75 \%$ of cases, with anorexia, weight loss, night sweats, and dyspnea. Ophthalmoscopy may detect typical choroidal tubercles in the retina. Almost one-third of the children with active TB may have extrapulmonary manifestations, such as adenitis (frequently as a nontender, firm-tender, firm cervical lymphadenitis, or TB meningitis (see Section 5.8.1).

The diagnosis of TB is established by:

- History of contact with an infectious case;

- Symptoms: persistent, unremitting cough, persistent fever and fatigue, night sweating, chest pain, and weight loss;

- Identification of the mycobacteria (positive in about 30-40\% of cases), from sputum, gastric fluid, pleural fluid, CSF, or other tissues, or by PCR. Acid-fast smear is positive in $10-20 \%$;

- X-ray findings, often in the form of "unresolved pneumonia", with enlarged mediastinal lymphadenopathy;

- Positive tuberculin test, performed by using 5 tuberculin units of purified protein derivative (PPD). A positive reaction is $5 \mathrm{~mm}$ or more induration present after $48-72 \mathrm{~h}$;

- Detection of Mycobacterium tuberculosis specific antigens (IFN- $\gamma$ release assay).

Fever in TB may occur in the following situations:

- In pulmonary (e.g. miliary TB) and extrapulmonary TB as a leading manifestation of the disease. Children with combined intrapulmonary and extrapulmonary TB have a higher peak and a longer duration of fever than those with intrapulmonary TB alone [66];

- In any form of TB as a persistent sign for several months, even after appropriate therapy has been instituted. Fever in excess of $38.8^{\circ} \mathrm{C}$ often correlates with persistent fever for more than 2 weeks, and sometimes up to several months. Persistent fever is mostly due to the disease itself;

- In HIV as a coinfection, often present as unresolving pneumonia. This carries a high mortality despite adequate anti-TB and HIV therapy;

- In hypersensitivity to anti-tuberculous drugs (usually appearing between the third and fifth day of treatment). This should be considered in any patient with persistent fever after initiation of therapy. Such a drug reaction should be suspected if the fever becomes higher than it was prior to therapy and when other manifestations of hypersensitivity such as rash or eosinophilia appear;

- In adult TB-infected patients in about $40 \%$, usually low-grade and appearing insidiously.

Drugs used for treatment of TB are shown in the Table 5.16. A 6-month regimen for drug-susceptible TB with isoniazid (INH) and rifampicin and pyrazinamide for the first 2 months followed by INH and rifampicin for the remaining 4 months is recommended. If drug resistance is possible, initial treatment should include ethambutol, streptomycin, amikacin, or ciprofloxacin until drug susceptibility result becomes available. Shorter regimes using four drugs in the initial phase are increasingly being adopted. 


\subsection{1 .2}

\section{Malaria}

Malaria is caused by a protozoan of the genus Plasmodium transmitted by anopheles mosquitoes. The four species that commonly infect man are $P$. malariae (benign quartan malaria), $P$. vivax, $P$. ovale (benign tertian malaria), and $P$. falciparum (malignant tertian malaria). Whereas $P$. vivax invades mostly the youngest erythroblast and $P$. malariae invades primarily the older erythrocytes, in both no more than $1-2 \%$ of erythrocytes are infected at a time. P. falciparum, on the other hand, invades all ages of erythrocytes indiscriminately, resulting in a very high infection rate. The number of malaria cases and deaths is estimated at 200-300 million and 2-3 million, respectively. Over $50 \%$ of childhood deaths in many parts of Africa are attributed to malaria. Most cases of imported malaria to the United Kingdom are P. falciparum and vivax.

Patients with $P$. falciparum infection have elevated TNF- $\alpha$, soluble IL-2 receptors, and natural killer cell activity, but a decrease in the CD4:CD8 lymphocyte ratio. The level of TNF correlates with the severity and mortality rate in patients with this infection. Changes in the TNF also correlate with the rise and fall in temperature during $P$. vivax paroxysms.

Infected children present with fever, lethargy, headache, cough, anorexia, nausea, vomiting, diarrhea, abdominal pain, and dehydration. Physical examination reveals splenomegaly (detected in almost 100\%) and commonly hepatomegaly. Nephrotic syndrome may occur with $P$. malariae infection. The presenting clinical signs of cerebral malaria are severe headache, irritability, delirium, coma, hyperpyrexia, convulsion, and meningism.

In endemic regions, malaria is a major cause of fever, occurring in virtually $100 \%$ of cases. Classical periodicity of fever may not occur in children during the first few years of life: intermittent, continuous, or remittent patterns may all occur.

A child with fever caused by malaria may present with the following:

- A typical tertian paroxysm ( $P$. vivax and $P$. ovale) in a nonimmune child (usually in the afternoon or evening) with shivering and rigor, lasting $1-2 \mathrm{~h}$. The skin is cold and pale. The next stage is marked by high fever, up to $41{ }^{\circ} \mathrm{C}$, lasting $2-4 \mathrm{~h}$. The skin is dry and warm and the patient feels hot and has usually headache. The last $2-4 \mathrm{~h}$ are characterized by a drop in body temperature to normal with sweating. In tertian infection, the paroxysm recurs at $48 \mathrm{~h}$ intervals (Fig. 5.4) while in quartan infection the paroxysm recurs at $72 \mathrm{~h}$ intervals (Fig. 5.5). Early in the infection with $P$. falciparum the fever may be irregular or continuous. A significant correlation exists between heavy parasitemia of $2 \%$ or greater and high grade temperature.

- Malaria is the most common cause of febrile seizure (FS) worldwide.

- Blackwater fever is a state of acute intravascular hemolysis accompanied by hemoglobulinuria, as a complication of $P$. falciparum.

- Human parvovirus B19 infection adds to the severity of anaemia. The virus is highly erythrotropic, infecting erythroid progenitor cells.

- Associated diseases as a complication of malaria are, for example, pneumonia, or anemia. 


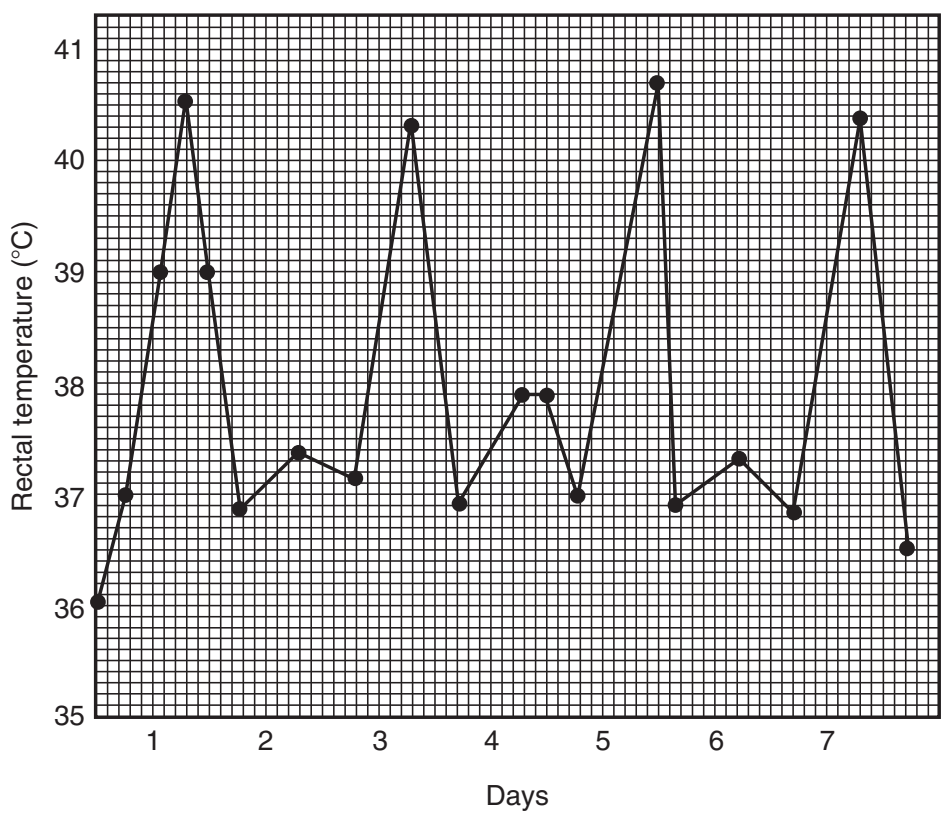

Fig. 5.4 Febrile cycle seen in tertian malaria caused by Plasmodium vivax infection

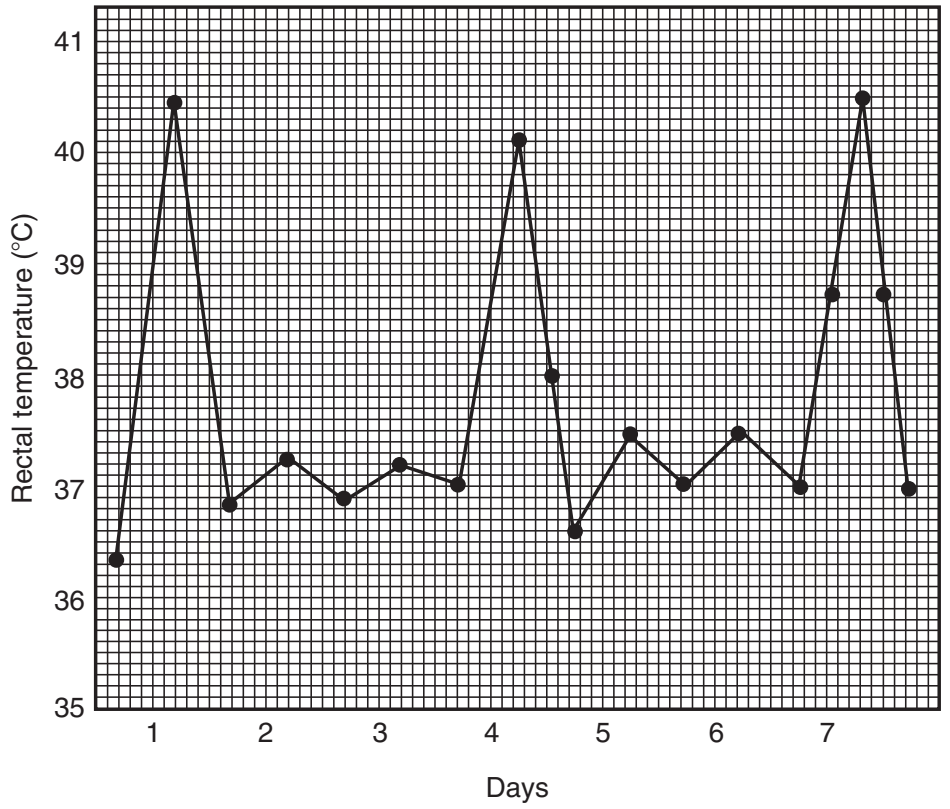

Fig. 5.5 Febrile cycle seen in quartan malaria caused by Plasmodium malariae infection 
Table 5.17 Drugs used for prophylaxis and therapy of malaria ${ }^{a}$

\begin{tabular}{|c|c|c|}
\hline & Dose & Adverse reaction \\
\hline \multicolumn{3}{|l|}{ Prophylaxis } \\
\hline Chloroquine & $5 \mathrm{mg}$ base $/ \mathrm{kg} /$ weekly & Retinal damage \\
\hline Fansidar & $125-750 \mathrm{mg}$ (according to the age) & Severe mucocutaneous reaction \\
\hline \multicolumn{3}{|l|}{ Therapy } \\
\hline Chloroquine & $\begin{array}{l}10 \mathrm{mg} \mathrm{kg}^{-1} \text {; followed by } 5 \mathrm{mg} \mathrm{kg}^{-1} \text { in } 6 \mathrm{~h} \text {, } \\
\text { then } 5 \mathrm{mg} \mathrm{kg}^{-1} \\
\text { Twice daily for } 2 \text { days }\end{array}$ & $\begin{array}{l}\text { Gastrointestinal upset, visual } \\
\text { disturbance, rash }\end{array}$ \\
\hline Quinine & $\begin{array}{l}25 \mathrm{mg} \mathrm{kg}^{-1} \text { per day three times for } 10-14 \\
\text { days }\end{array}$ & Tinnitus \\
\hline Primaquine & $0.3 \mathrm{mg}$ base $\mathrm{kg}^{-1}$ once daily for 14 days & $\begin{array}{l}\text { Methaemoglobulinemia, haemo- } \\
\text { lytic anemia (in G6PD) }\end{array}$ \\
\hline
\end{tabular}

a Treatment of malaria varies considerably around the world and advice about antimalarials will depend upon the location and expert advice

- The pattern of fever is irregular (and not with typical periodic fever). This may occur in young children in early falciparum infection or as a consequence of previous chemoprophylaxis, which modifies the typical pattern of fever.

- PUO is present, with fever as the only sign of malaria without anemia or splenomegaly.

- Recurrent fever months after treatment of falciparum malaria persist owing to relapsing vivax or ovale infection if the child initially had a mixed infection [67].

Diagnosis is easy when children present with typical paroxysms of fever. Definite diagnosis is made by a Giemsa-stained blood smear (thick smear increases the yield). A rapid diagnosis can be obtained by utilizing ribosomal ribonucleic acid (rRNA) of the parasite. Laboratory findings include anaemia, with $\mathrm{Hb}$ concentration of $5-11 \mathrm{~g} \mathrm{dl}^{-1}$ and leucopenia. Thrombocytopenia, hyponatremia, and hypoglycemia may also occur.

Therapy. Hospitalization for any child with suspected or confirmed malaria is always indicated to assess severity and extent of severity. Paracetamol is commonly used for fever management. Paracetamol has been reported to prolong parasitemia, although the evidence for that was found to be insufficient [68]. Chemoprophylaxis and therapy are shown in the Table 5.17. Chloroquine remains the treatment for choice for benign malarias, while quinine is given for falciparum malaria. Children receiving iron therapy may be at increased risk of fever associated with severe parasitemia (see the section on anaemia in Chapter 6)

\subsection{1 .3 \\ Brucellosis}

Brucellosis is primarily a zoonotic infection caused by small, nonmotile, Gram-negative coccobacilli of the genus Brucella. There are four important species pathogenic to humans: B. melitensis (Malta fever, found primarily in goats and sheep), B. abortus (abortus fever, in cattle), B. suis (swine), and B. canis (dogs). The infection is transmitted to humans through direct contact with infected animals or their products, and through consumption 
of infected milk, milk products, or meat. More than half a million cases per year occur worldwide.

The clinical features of brucellosis depend largely upon the infected species of organism. Infection with $B$. melitensis produces more severe symptoms and signs than other species, with fever, arthritis, arthralgia, backache, anorexia and weight loss, tender hepatosplenomegaly, and lymphadenopathy.

\section{Fever is a prominent feature in brucellosis, which manifests:}

- in almost every patient ( $90-100 \%$ of cases);

- often insidiously over the course of several days;

- sometimes suddenly with chills, rising sharply to a peak up to $40.5^{\circ} \mathrm{C}$ and swinging considerably;

- as a remittent pattern, falling usually to a normal level;

- as periodic fever, with symptoms lasting a few days or weeks followed by symptomfree intervals that last weeks and months. During this period patients feel tired and may run a low fever in the evening; and

- as PUO, particularly in endemic areas.

Complications include spondylitis, osteomyelitis, granulomatous reaction of the eye, meningitis, or meningo-encephalitis. B. endocarditis is rare and may be responsible for most death due to the disease

Laboratory findings include anemia, leucopenia, lymphopenia, and raised liver enzymes. The diagnosis is established by positive culture of brucella organisms from blood or bone marrow aspirate, or positive serological tests (agglutination titer of $>1: 80$ ).

Several antibiotics are recommended. In a multicenter therapeutic study of 1100 children with brucellosis in Kuwait (101), the most effective treatment in young children was a combination Co-trimoxazole for 3 weeks and gentamicin during the first 5 days. In children older than 8 years, a combination of doxycycline (or tetracycline) is given for three weeks and gentamicin during the first 5 days. Rifampicin has also been used successfully in combination with streptomycin in the treatment of brucella endocarditis.

\subsection{1 .4}

\section{Lyme Disease}

Lyme disease (LD) is a multisystem inflammatory disease caused by the spirochete Borerlia burgdorferi. It is transmitted by the deer tick (Xodides dammini). LD is the most common vectorborne illness in the United States. B. burgdorferi is a potent inducer of IL-1 from peripheral blood mononuclear cells.

Lyme disease has been divided into three stages:

- The first one consists of a flu-like illness with a characteristic annular skin rash (erythema migrans), which develops at the site of the tick bite in approximately twothirds of patients. Antibiotics at this stage may prevent subsequent stages.

- The second stage follows 2-12 weeks after the tick bite and is characterized by disseminated infection causing aseptic meningitis and cranial neuritis (most commonly 
presenting as Bell palsy) and carditis (most commonly presenting as atrioventricular block or myocarditis).

- The third stage is characterized by oligoarticular arthritis and acrodermatitis chronica atrophicans from 6 weeks to 2 years after the tick bite in $50-80 \%$ of patients.

IL-1 and IL-1 receptor antagonist, both produced by monocytes and macrophages, may influence the course of arthritis. High concentration of IL-1 receptor antagonist and low concentration of IL-1 may indicate rapid resolution of arthritis, whereas the reverse pattern of cytokine concentration may indicate recovery.

Fever is often an early of the disease, occurring with other flu-like manifestations. Fever is usually intermittent and low-grade and has been reported in about $50 \%$ of children. Fever, however, can be as high as $40^{\circ} \mathrm{C}$ and persistent, which can cause PUO.

Diagnosis of LD depends on characteristic clinical features, in particular the appearance of erythema migrans. Specific IgM antibodies against B. burgdorferi appear 3-4 weeks after the infection and peak after $6-8$ weeks. Specific IgG antibodies usually become detectable in the second month after the onset of infection.

Uncomplicated cases of LD are treated with oral penicillin or amoxicillin divided into three doses for 21 days. For children older than 12 years, doxycycline twice daily for 21 days or tetracycline for the same duration is effective. For arthritis, antibiotic therapy should continue for 4 weeks and often includes a third-generation cephalosporin. For meningitis, penicillin $\mathrm{G} /$ cephalosporin is given intravenously for $2-3$ weeks.

\subsection{1 .5}

\section{Leptospirosis}

Leptospirosis is a zoonosis caused by the genus Leptospira. Human infections occur through contact with water (e.g., flood water) or soil contaminated with infected animal's urine. The incubation period is usually between 6 and 12 days. The disease is characteristically biphasic:

- The primary phase manifests as an influenza-like illness lasting 5-7 days with abrupt onset of chills and fever $39.5-40.5^{\circ} \mathrm{C}$ lasting 3-7 days. This phase reflects the presence of leptospiremia.

- In the second phase, lasting 4-30 days, specific antibodies begin to appear. Fever is not prominent in this phase, but may occur as a result of aseptic meningitis mediated by antigen-antibody reaction.

Clinically, the patients presents in one of the following three forms:

- The mild and more common form of the disease is characterized by headache, myalgia, arthralgia, abdominal pain, and conjunctival suffusion. Fever lasts longer than 5 days in two-thirds of cases.

- In the more severe and potentially fatal form, patients present with jaundice, hemorrhage, anemia, disturbance of consciousness, and renal dysfunction.

- PUO may occur in about $10 \%$ of cases.

Laboratory findings include leukocytosis, hyperbilirubinemia, and intravascular hemolysis. Leptospirochets may be cultured from the blood (dark-field microscopy), urine, and CSF. 
IgM-ELISA test may confirm the diagnosis. Penicillin is the treatment of choice and should be given during the first phase of illness to prevent complications.

\subsection{6 \\ Leishmaniasis}

Of the three clinical forms of leishmaniasis, cutaneous, mucocutaneous, and visceral, only the latter form is associated with febrile episodes. The disease is transmitted to humans by bite of infected sandfly Phlebotomus. It is the second most fatal parasitic disease after malaria. Coinfection with HIV has been reported [69].

The visceral form (also known as kala azar, black fever) is caused by the protozoan Leishmania donovani. The infection produces the following clinical course:

- Following the invasion of the blood stream, the organisms settle in the reticuloendothelial system and viscera, where they multiply within the cell's cytoplasm despite being engulfed by mononuclear cells. Eventually, the mononuclear cells rupture and release many organisms, which are subsequently engulfed by other phagocytic cells.

- A few months after the initial bite, the patients develop symptoms manifested by varying degree and patterns of fever (see below), emaciation, massive hepatosplenomegaly, lymphadenopathy, profound weakness, and pancytopenia. The weakness is mainly caused by anemia and chronic infection. The pancytopenia is caused by a combination of invading the bone marrow by leishmania, hypersplenism, and autoimmune process. Thrombocytopenia may be severe enough to produce bleeding. Leukopenia causes secondary bacterial infections, such as pneumonia.

- Death occurs within 1-2 years in $80-90 \%$ of untreated patients.

All infected children have fever (Table 5.18), which manifests in protean patterns:

- In young children, it increases gradually to a peak within 2 weeks from the onset $\left(40-41.1^{\circ} \mathrm{C}\right)$, becoming then intermittent (temperature returning to a normal level within the same day) or continuous (fever fluctuates by less than $1^{\circ} \mathrm{C}$ ) and resolving usually by lysis.

\begin{tabular}{ll} 
Clinical findings & $\%$ \\
\hline Fever & 100 \\
Splenomegaly & 96 \\
Hepatomegaly & 91 \\
Abdominal distention & 89 \\
Respiratory distress & 50 \\
Diarrhea & 13 \\
Jaundice & 3 \\
Associated septicemia & 2 \\
Hemorrhage & 1 \\
\hline
\end{tabular}

Table 5.18 symptoms and signs of kala azar in 100 children, admitted to Baghdad's University Hospital 
- In older children, with more chronic presentation, fever may be continuous initially but is usually low grade.

- Classically fever is double quotidian (two spikes within $24 \mathrm{~h}$ or a $12 \mathrm{~h}$ cycle).

- PUO may occur with a duration between 1 and 18 months [70], with a median duration of fever of 4-5 weeks.

Pathogenesis and fever are related to the interaction of T-helper cells and various cytokines. INF- $\gamma$, IL-6, and IL-4 are involved during active disease [71]. IL-10 is known to suppress the macrophages, which may explain the paucity of cytokines secreted by these cells. Diagnosis is by identifying the parasite from bone marrow or splenic aspirate. Treatment is presently with Miltefosine, orally $2.5 \mathrm{mg} \mathrm{kg}^{-1}$ per day for 4 weeks. Pentavalent antimony compounds and liposomal amphotericin B are also used. Defervescence usually occurs after a median of 6 days of treatment.

\subsection{1 .7}

\section{Fever and Malnutrition}

It has been estimated that 10.6 million children are still dying yearly, mostly due to pneumonia, diarrhea, neonatal causes and, in sub-Saharan Africa, malaria [72]. Malnutrition was an underlying cause in over $50 \%$ of the deaths. These children are particularly susceptible to measles and tuberculosis. Chronic infections, on the other hand, may lead to loss of nutrients and malnutrition.

Protein-calorie malnutrition (PCM) has been divided into severe (kwashiorkor, marasmus, and intermediate cases), moderate severe (nutritional, dwarfing, or stunting and wasting), and early (clinically detectable only by anthropometric measurement). In developing countries, the severe form of malnutrition is common as a result of several factors, including severe dietary imbalance. Marasmus results from deficiency of all nutrients, whereas kwashiorkor is due primarily to protein deficiency. In developed countries, PCM may result from debilitating chronic diseases.

Patients with PCM are susceptible to infection and fever for a variety of reasons:

- Delayed mononuclear cell release from the bone marrow

- Impaired T-cell-mediated immunity, deficiency in circulating levels of the complement system and interferon

- Normally, reduced secretary IgA (B cells and circulating immunoglobulins)

- Impaired acute-phase response

Fever in a malnourished child is usually the result of infection. Respiratory infection, for example, pneumonia, TB, and intestinal infection are most common. In severe malnutrition, fever may be absent, and instead hypothermia may occur in response to infection signifying a poor prognosis for survival.

Malnourished children who are febrile are at increased risk of paracetamol-induced hepatotoxicity. Reduction in calorie or protein intake in association with multiple doses of paracetamol may have profound effects on sulfate and glucuronide. The combination of malnutrition and HIV is particularly devastating. 


\section{References}

\section{Acute URTI}

1. Dingle JH, Badger GF, Jordan WS. Illness in the home. Press of Western Reserve University. Cleveland, Ohio 1964

2. Scott GM, Secher DS, Flowers D, et al. Toxicity of interferon. Br Med J 1981; 282: 1345-8

3. Putto A, Ruuskanen O, Meurman O. Fever in respiratory virus infection. Am J Dis Child 1986; 140: 1159-63

4. Wright PF, Ross KB, Thompson J, et al. Influenza A infections in young children: Primary natural and protective efficacy of live-vaccine-induced or naturally acquired immunity. N Engl J Med 1977; 296: 829-34

\section{Infectious Mononucleosis}

5. Shurin S. Infectious mononucleosis. Pediatr Clin North Am 1979; 26: 315-26

6. Rapp CE, Hewetson JF. Infectious mononucleosis and the Epstein-Barr virus. Am J Dis Child 1978; 132: 78-86

7. Auwaerter PG. Recent advance in the understanding of infectious mononucleosis: Are prospects improved for treatment or control? Expert Rev Anti Infect Ther 2006; 4: 1039-49

\section{Acute Upper Airway Obstruction}

8. Davis HW, Carter JC, Galvis AG, et al. Acute upper airway obstruction: Croup and epiglottitis. Pediatr Clin North Am 1981; 28: 859-80

\section{Bronchiolitis \& Asthma}

9. El-Radhi AS, Barry W, Patel S. Association of fever and severe clinical course in bronchiolitis. Arch Dis Child 1999; 81: 231-4

10. El-Radhi AS, Carroll J. Fever and hyperthermia. In: Fever in paediatric practice. Blackwell. Oxford 1994, p.19

11. Issac D. Production of interferon in respiratory syncytial virus bronchiolitis. Arch Dis Child 1989; 64: 92-5

12. El-Radhi AS, Patel S. The Clinical Course of Childhood Asthma in Association with Fever (Unpublished)

13. El-Radhi AS, Hogg CL, Bungre JK, et al. Effect of oral glucocorticoid treatment on serum inflammatory markers in acute asthma. Arch Dis Child 2000; 83: 158-62

14. Philips K, Shikany S. The value of hyperpyrexia in the treatment of asthma. South Med J 1935; 28: 801-12

15. Nickels DA, Moore DC. Serum cortisol responses in febrile children. Pediatr Infect Dis J 1989; 8: 16-20 


\section{Pneumonia}

16. Levin S. The atypical pneumonia syndrome. J Am Med Assoc 1984; 251: 945-8

17. McCarthy PL, Tomasso L, Dolan TF. Predicting fever response pf children with pneumonia treated with antibiotics. Clin Pediatr 1980; 19: 753-60

18. Clark JE, Hammal D, Spencer D, et al. Children with pneumonia: How do they present and how are they managed?. Arch Dis Child 2007; 92: 394-8

19. Copps S, Allen V, Sueltmann S, et al. Community-outbreak of mycoplasma pneumonia. J Am Med Assoc 1968; 204: 121-6

20. Hughes WT, Price RA, Kim HK, et al. Penumonitis in children with malignancies. J Pediatr 1973; 82: 404-15

\section{Gastroenteritis}

21. William CH, Robert HG, Robert EB, et al. Effects of nutritional status on diarrhea in Peruvian children. J Pediatr 2002; 140: 210-6

22. Kosek M,Bern C, Guerrant RL. The global burden of diarrhoeal disease, as estimated from studies published between 1992 and 2000. Bull World Health Organ 2003; 81(3): 197-204

23. Bhutta ZA, Belgaumi A, Abdur Rab M, et al. Child health and survival in the Eastern Mediterranean region. Br Med J 2006; 333: 839-42

24. Glaeson M, Merson MH. Global progress in the control of diarrhoeal diseases. Pediatr Infect Dis J 1990; 9: 345-55

25. Vargas M, Gascon J, Casals C, et al. Etiology of diarrhea in children less than five years of age in Ifakara, Tanzania. Am. J. Trop. Med. Hyg. 2004; 70(5): 536-9

26. Vu Nguyen T, Le Van P, Le Huy C, et al. Etiology and epidemiology of diarrhea in children in Hanoi, Vietnam. Int J Infect Dis 2006; 10: 298-308

27. Klein EJ, Boster DR, Stapp JR, et al. Diarrhea etiology in a Children's Hospital Emergency Department: A prospective cohort study. Clin Infect Dis 2006; 43(7): 807-13

28. Kariuki S, Revathi G, Kariuki N, et al. Characterisation of community acquired non-typhoidal Salmonella from bacteraemia and diarrhoeal infections in children admitted to hospital in Nairobi, Kenya. BMC Microbiol. 2006; 6: 101

29. Chalker PR, Blaser MJ. A review of human salmonellosis: Magnitude of salmonella infection in the United States. Rev Infect Dis 1988; 10: 111-23

30. Stoycheva M, Murdjeva M. Serum levels on INF-gamma, IL-12, TNF-alpha, and IL-10, and bacterial clearance in patients with gastroenteric Salmonella infection. Scand J Infect Dis 2005; 37: 11-14

31. El-Radhi AS, Rostila T, Vesikari T. Association of high fever and short bacterial excretion after salmonellosis. Arch Dis Child 1992; 67: 531-2

32. Ryan CA, Hargrett-Bean NT, Blake PA. Salmonella typhi infections in the United States 1975-1984: Increasing role of foreign travel. Rev Infect Dis 1989; 11: 1-9

33. El-Radhi AS, Newcombe T, Ghalli A. Effect of pyrexia on Shigella and Salmonella gastroenteritis (unpublished)

34. Hiranrattana A, Mekmuullica J, Chatsuwan T, et al. Childhood shigellosis at King Chulalongkorn Memorial Hospital, Bangkok, Thailand: A 5-year review (1996-2000). Southeast Asian J Trop Med Public Health. 2005; 36(3): 683-5

35. Thoren A, Wolde-Mariam T, Stintzing G, et al. Antibiotics in the treatment of gastroenteritis caused by enteropathogenic Escherichia coli. J Infect Dis 1980; 141: 27-31 
36. Mohammed HF, Hassan MK, Bakir SS. Campylobacter jejuni Gastroenteritis in children in Basrah, Iraq. Med J Basrah Univ 2004; 22(1-2): 1-5

37. Marks MI, Pai CH, Lafleur L, et al. Yersinia enterocolitica: A prospective study of clinical, bacteriologic, and epidemiologic features. J Pediatr 1980; 96: 26-31

38. Cook SM, Glass RI, Le Baron CW. Global seasonality of Rota virus infection. Bull World Health Organ 1990; 68: 171-177

39. Blutt SE, Matson DO, Crawford SE, et al. Rotavirus Antigenemia in Children Is Associated with Viremia. PLoS Med. 2007; 4: e121

40. Ruuska T, Vesikari T. Rotavirus disease in Finnish children: Use of numerical scores for clinical severity of diarrhoeal episodes. Scand J Infect Dis 1990; 22: 259-267

41. Brandt CD, Rodriguez WJ, Arrobio JO, et al. Adenovirus and pediatric gastroenteritis. J Infect Dis 1985; 151: 437-43

42. Grajden M, Brown M, Petrasek A, et al. Clinical features of adenovirus gastroenteritis: A review of 127 cases. Pediatr Infect Dis J 1990; 9: 636-41

43. Rautanen T, El-Radhi AS, Vesikari T. Clinical experience with a hypotonic oral rehydration solution in acute diarrhoea. Acta Paediatr 1992; 81: 1-3

44. BMJ Publishing Group.Clinical evidence. BMJ Publishing Group. UK 2000; 4th Issue, pp. 373-9

UTI

45. Ginsburg CM, McCracken GH. Urinary tract infection in young children. Pediatrics 1982; 69: 409-12

46. Zorc JJ, Levene DA, Platt SL, et al. Clinical and demographic factors associated with UTI in young febrile infants. Pediatrics 2005; 116: 644-8

47 Hodson EM, Willis NS, Craig JC. Antibiotics for acute pyelonephritis in children. Cochrane Database Syst Rev 2007; no 4p. CD003772

HIV

48. Steinbrook R. Message from Toronto-Deliver AIDS treatment and prevention. N Engl J Med 2006; 355: 1081-4

49. Greenberg AE, Dabis F, Marum LH, de Cock KM. HIV infection in Africa. In, Pizzo PA, Wilfert CM (Eds). Pediatric AIDS. The challenge of HIV infection in infants, children and adolescents. 3rd Edition. Lippincott. Philadelphia. 1998; pp. 23-46

50. Andiman WA, Mezger J, Shapiro E. Invasive bacterial infections in children born to women infected with human immunodeficiency virus type 1. J Pediatr. 1994; 124: 846-52

51. Ruiz-Contreras J, De Jose MI, Ciria L, Mellado MJ, Ramos JT, Clemente J, Rodriguez-Cerrato V. Fever of unknown origin in HIV infected children. Int Conf AIDS. 1998; 12: 44 (abstract no. 12165)

\section{Infection of the CNS}

52. Dery MA, Hasbun R. Changing epidemiology of bacterial meningitis. Curr Infect Dis Rep 2007; 9: 301-7 
53. Holt DE, Halket S, de Louvois J, et al. Neonatal meningitis in England and Wales: 10 years on. Arch Dis Child Fetal Neonatal Ed 2001; 84: F85-9

54. Thompson MJ, Ninis N, Perera R, et al. Clinical recognition of meningococcal disease in children and adolescent. Lancet 2006; 367: 397-403

55. Heckmatt JZ. Coliform meningitis in the newborn. Arch Dis Child 1976; 51: 569-73

56. Wong VK, Hitchcock W, Mason WH. Meningococcal infection in children: A review of 100 cases. Pediatr Infect Dis J 1989; 8: 224-7

57. Small PM, Täuber MG, Hackbarth CJ, et al. Influence of body temperature on bacterial growth rate in experimental pneumococcal meningitis in rabbits. Infect Immun 1986; 52: 484-7

58. Sande MA, Sande ER. The influence of fever on the development of experimental Streptococcus pneumoniae meningitis. J Infect Dis 1987; 156: 849-50

59. Lin TY, Nelson JD, McCracken GH. Fever during treatment for bacterial meningitis. Pediatr infect Dis 1984; 3: 319-22

60. Rantala H, Uhari N. Occurrence of childhood encephalitis: A population-based study. Pediatr Infect Dis 1989; 8: 426-30

61. Koskiniemi M, Vaheri A. Acute encephalitis of viral origin. Scand J Infect Dis 1982; 14: $181-7$

62. Saez-Liorens XJ, Umana MA, Odio CM, et al. Brain abscess in infants and children. Pediatr Infect Dis 1989; 8: 449-58

\section{Osteomyelitis}

63. Ceroni D, Requsci M, Pazos J, et al. Acute bone and joint infection in children: How much attention should be paid to persistent fever during IV antibiotic therapy. Rev Chir Orthop Repar Appar Mot 2003; 89: 250-6

\section{Viral Exanthems}

64. Miller CL. Current impact of measles in the United Kingdom. Rev Infect Dis 1983; 5: $427-38$

\section{Tropical Diseases}

65. Snider DE, Rieder HL, Combs D, et al. Tuberculosis in children. Pediatr Infect Dis J 1988; 7 : 271-8

66. Lin YS, Chering HY, Yin CL. Clinical characteristics of tuberculosis in children in the North of Taiwan. J Microb Immun Infect 2005; 38: 41-6

67. Brabin JB Ganley Y. Imported malaria in children in the UK. Arch Dis Child 1997; 77: $76-81$

68. Meremikkwu M, Logan K, Garner P. Antipyretic measures for treating fever in malaria. The Cochrane Database Syst Rev 2000, Issue 2. Art. No.: CD002151. D0I: 10.1002/14651858. CD002151

69. Mathur P, Samantaray JC, Vajpayee M, et al. Visceral leishmaniasis/HIV co-infection in India. J Med Microbiol 2006; 55: 919-22 
70. Mathur P, Samantaray J, Chauhan NK. Evaluation of a rapid immunochromatographic test for diagnosis of kala azar and post kala azar dermal leishmaniasis at a tertiary care centre of north India. Indian J Med Res 2005; 122: 485-90

71. Ansari NA, Saluia S, Salotra P. Elevated levels of INF- , IL-10 and IL-6 during active disease in Indian kala azar. Clin Immunol 2006; 119: 339-45

\section{Fever in Malnutrition}

72. Bryce J, Boschi-Pinto C, Shibuya K, et al. World Health Organisation estimate of the causes of death in children. Lancet 2005; 365: 1147-52 